\title{
Systematic investigation of low-lying dipole modes using the canonical-basis time-dependent Hartree-Fock-Bogoliubov theory
}

\author{
Shuichiro Ebata, 1,2,3 Takashi Nakatsukasa, ${ }^{3,4}$ and Tsunenori Inakura ${ }^{3,5}$ \\ ${ }^{1}$ Meme Media Laboratory, Hokkaido University, Sapporo, 060-0813, Japan \\ ${ }^{2}$ Center for Nuclear Study, University of Tokyo, Wako-shi 351-0198, Japan \\ ${ }^{3}$ RIKEN Nishina Center, Wako-shi 351-0198, Japan \\ ${ }^{4}$ Center for Computational Sciences, University of Tsukuba, Tsukuba 305-8571, Japan \\ ${ }^{5}$ Department of Physics, Graduate School of Science, Chiba University, Chiba, 263-8522, Japan
}

(Received 28 March 2014; published 7 August 2014)

\begin{abstract}
Systematic investigations of the electric dipole $(E 1)$ modes of excitation are performed using the canonicalbasis time-dependent Hartree-Fock-Bogoliubov (Cb-TDHFB) theory. The Cb-TDHFB is able to describe dynamical pairing correlations in excited states of nuclear systems. We apply the method to the real-time calculation of linear response in even-even nuclei with Skyrme functionals. Effects of shell structure, neutron skin, deformation, and neutron chemical potential (separation energy) are studied in a systematic way. This reveals a number of characteristic features of the low-energy $E 1$ modes. We also find a universal behavior in the low-energy $E 1$ modes for heavy neutron-rich isotopes, which suggests the emergence of decoupled $E 1$ peaks beyond $N=82$.
\end{abstract}

DOI: 10.1103/PhysRevC.90.024303

PACS number(s): 21.10.Pc, 21.60.Jz, 24.30.Gd, 25.20.-x

\section{INTRODUCTION}

Elementary modes of excitation provide us with fundamental information on quantum many-body systems. A novel structure in the ground state may lead to a new type of elementary excitations. In studies of radioactive isotopes, the low-energy electric dipole $(E 1)$ modes of excitation, pygmy dipole resonance (PDR), are currently of significant interest.

The giant dipole resonance (GDR) at high frequency is a fundamental mode of excitation in finite nuclei. The GDR is universally observed in all nuclei, from light to heavy, from stable to unstable isotopes, and exhausts almost $100 \%$ of the energy-weighted sum rule (EWSR) value. It is also known to reflect bulk properties of nuclei, such as the symmetry energy. In contrast, the PDR is sensitive to the structure of an individual nucleus. Since most of the $E 1$ strength is carried by the GDR, the $E 1$ strength of low-energy states is known to be significantly hindered. The presence of substantial $E 1$ strength in the PDR implies that the structure of the nucleus is significantly different from our conventional understanding. Thus, it is important to identify the basic properties and the origin of the PDR.

The low-energy dipole states have been observed in a number of nuclei: neutron-rich $\mathrm{O}$ isotopes $[1,2],{ }^{26} \mathrm{Ne}$ [3], stable $\mathrm{Ca}$ isotopes [4,5], ${ }^{56} \mathrm{Fe}$ and ${ }^{58} \mathrm{Ni}[6],{ }^{68} \mathrm{Ni}[7],{ }^{88} \mathrm{Sr}[8]$, ${ }^{90} \mathrm{Zr}$ [9,10], $\mathrm{Sn}$ isotopes [11-15], $N=82$ isotones [16-21], and stable $\mathrm{Pb}$ isotopes [22-26]. The observed low-energy $E 1$ strength exhausts less than $1 \%$ of the Thomas-Reiche-Kuhn (TRK) sum-rule value in stable isotopes, while it may amount up to about $5 \%$ in neutron-rich nuclei.

Theoretically, the PDR has been commonly investigated using the (quasiparticle) random phase approximation [(Q)RPA] [27-43]. There are some calculations beyond the (Q)RPA as well [44-49]. However, even in the (Q)RPA level, different models often predict different properties. The nature of the PDR is still elusive with a lack of general agreement.
In order to clarify properties of the PDR, we think it important to study a variety of isotopes systematically, from light to heavy, from spherical to deformed, and from normal to superfluid nuclei. Using a technique of the finite amplitude method [50-56], a systematic investigation for the PDR has been carried out for nuclei up to $Z=40$ in Ref. [57], in which the pairing correlations were neglected. In this article, we report an analysis extended to a heavier region, taking into account the pairing correlations. To perform the study, we utilize the canonical-basis time-dependent HartreeFock-Bogoliubov (Cb-TDHFB) method [29]. We carry out a systematic calculation for the $E 1$ modes of excitation, with a parallelized computer code for the real-time evolution of the Cb-TDHFB equations in the three-dimensional (3D) coordinate-space representation. The method is able to take a full account of nuclear deformation of any kind, and treat the pairing correlation in a BCS-like scheme, both in static and in dynamic natures. The numerical cost of the $\mathrm{Cb}$-TDHFB method is significantly smaller than that of the normal QRPA calculations [29], which enables us to perform a systematic analysis for nuclei in a wide region of the nuclear chart. However, since the BCS-like treatment of the pairing correlation leads to the neutron gas problem very near the drip line [58], we limit our studies to nuclei with neutron separation energies larger than $2.0 \mathrm{MeV}$.

The nature of the low-lying modes of excitation in nuclei is still an open issue. Especially, the $E 1$ modes in neutron-rich nuclei attract much attention with a simple geometrical picture that the excess neutrons in the nuclear surface region oscillate against the core of the nucleus. This interpretation of the PDR as the "neutron-skin mode" was originally discussed using hydrodynamical models [59-61]. Then, naturally, one may expect that the collectivity of the low-energy $E 1$ transitions has a certain connection to a property of the excess neutrons and the neutron skin thickness. The correlation between the $E 1$ collectivity of PDR and the neutron skin thickness has been 
microscopically studied using the (Q)RPA [34,57,62]. Similar analysis has been performed for the correlation between the $E 1$ collectivity of PDR and the isovector nuclear matter properties, such as the symmetry energy $[14,42,62,63]$. The current situation is that, although everybody agrees with the existence of certain correlation among them, it is not well settled yet how strong the correlation is.

In this paper, we carry out systematic linear-response calculations for the $E 1$ mode for over 300 kinds of nuclei. Properties of the PDRs are investigated in terms of their isotopic dependence as a function of neutron number, their relation to the neutron skin thickness and separation energy, and the deformation effect.

The paper is organized as follows. In Sec. II, we present the $\mathrm{Cb}$-TDHFB method and real-time calculation of the $E 1$ strength functions. The definition of PDR fraction to quantify the low-lying strength is given, and the model-space dependence of the numerical results is examined. In Sec. III, we show variation of the PDR strengths with respect to the neutron number and the neutron-skin thickness, for nuclei in a wide mass region with $Z \leqslant 50$. We also discuss the functional dependence of the PDRs in Sn isotopes with the Skyrme parameter sets $\mathrm{SkM}^{*}$ and SkI3. Section IV presents the deformation effects on the PDR. The $K=0$ dominance of the PDR fraction is observed for nuclei with prolate shapes. We examine its relevance to the orientation dependence of the neutron-skin thickness. In Sec. V, we discuss the PDRs of heavy isotopes around $N=82$. The present study suggests that the existence of PDR peaks is not hindered by the coupling to GDR. Finally, the conclusion is given in Sec. VI.

\section{FORMULATION AND NUMERICAL DETAILS}

In this section, we recapitulate the $\mathrm{Cb}$-TDHFB method for the calculation of strength functions [29]. Let us first express the normal and pair densities, $\left(\rho_{\mu \nu}(t), \kappa_{\mu \nu}(t)\right)$, in terms of canonical pairs of states, $\left(\phi_{k}(t), \phi_{\bar{k}}(t)\right)$. Here, the indices $\mu, v$ mean arbitrary single-particle basis:

$$
\begin{aligned}
& \rho_{\mu \nu}(t)=\sum_{k} \rho_{k}(t)\left\{\left\langle\mu \mid \phi_{k}(t)\right\rangle\left\langle\phi_{k}(t) \mid \nu\right\rangle+\left\langle\mu \mid \phi_{\bar{k}}(t)\right\rangle\left\langle\phi_{\bar{k}}(t) \mid \nu\right\rangle\right\}, \\
& \kappa_{\mu \nu}(t)=\sum_{k} \kappa_{k}(t)\left\{\left\langle\mu \mid \phi_{k}(t)\right\rangle\left\langle\nu \mid \phi_{\bar{k}}(t)\right\rangle-\left\langle\nu \mid \phi_{k}(t)\right\rangle\left\langle\mu \mid \phi_{\bar{k}}(t)\right\rangle\right\},
\end{aligned}
$$

where $\rho_{k}(t)$ and $\kappa_{k}(t)$ are the occupation and the pair probabilities, respectively, which can be written as $\rho_{k}(t)=\left|v_{k}(t)\right|^{2}$ and $\kappa_{k}(t)=u_{k}(t) v_{k}(t)$ using the time-dependent $(u, v)$ coefficients in the canonical representation [64]. All we need to calculate are the time evolutions of $\phi_{k}(t), \phi_{\bar{k}}(t), \rho_{k}(t)$, and $\kappa_{k}(t)$. Equations to determine their time evolutions will be given in the following. Note that the density operators, $\left(\rho_{\mu \nu}, \kappa_{\mu \nu}\right)$, in the left-hand side of Eqs. (1) and (2) are matrices with two indices, while $\left(\rho_{k}, \kappa_{k}\right)$ in the right-hand side have a single index.

\section{A. Energy density functional}

The energy density functional is given by the sum of the Skyrme density functional, $E_{\text {Sky }}[\rho]$, and the pairing energy functional, $E_{\text {pair }}\left[\kappa, \kappa^{*}\right]$.

$$
E_{\mathrm{tot}}\left[\rho(t), \kappa(t), \kappa^{*}(t)\right]=E_{\mathrm{Sky}}[\rho(t)]+E_{\mathrm{pair}}\left[\kappa(t), \kappa^{*}(t)\right] .
$$

For the Skyrme energy functional, the $\mathrm{SkM}^{*}$ parameter set is used, unless otherwise specified. For the pairing part, we adopt a simple functional of the form

$$
\begin{aligned}
E_{\text {pair }}\left[\kappa(t), \kappa^{*}(t)\right] & =-\sum_{\tau=n, p} \sum_{k, l>0} G_{k l}^{\tau} \kappa_{k}^{\tau *}(t) \kappa_{l}^{\tau}(t) \\
& =-\sum_{\tau=n, p} \sum_{k>0} \kappa_{k}^{\tau *}(t) \Delta_{k}^{\tau}(t),
\end{aligned}
$$

where the gap parameter $\Delta_{k}(t)$ are given by

$$
\Delta_{k}^{\tau}(t)=\sum_{l>0} G_{k l}^{\tau} \kappa_{l}^{\tau}(t)
$$

Here, $G_{k l}^{\tau}=g^{\tau} f\left(\varepsilon_{k}^{0}\right) f\left(\varepsilon_{l}^{0}\right)$, and the constant $g$ is determined by the smoothed pairing method [65]. The cutoff function $f\left(\varepsilon_{k}^{0}\right)$ depends on the single-particle energy of the canonical state $k$ at the Hartree-Fock plus BCS (HF+BCS) ground state. The $f(\varepsilon)$ is written as

$$
f(\varepsilon)=\left(1+\exp \left[\frac{\varepsilon-\epsilon_{\mathrm{c}}}{0.5 \mathrm{MeV}}\right]\right)^{-1 / 2} \theta\left(e_{\mathrm{c}}-\varepsilon\right),
$$

with the cutoff energies

$$
\epsilon_{\mathrm{c}}=\tilde{\lambda}+5.0 \mathrm{MeV}, \quad e_{\mathrm{c}}=\epsilon_{\mathrm{c}}+2.3 \mathrm{MeV},
$$

where $\tilde{\lambda}$ is the midpoint of the highest occupied level and the lowest unoccupied level in the Hartree-Fock (HF) state. Here, the cutoff parameter $e_{\mathrm{c}}$ is necessary to prevent occupation of spatially unlocalized single-particle states. For neutrons, if $e_{\mathrm{c}}$ becomes positive, we replace it by zero [66]. Because of this limitation of the $\mathrm{HF}+\mathrm{BCS}$ calculation, we restrict our study to nuclei with neutron separation energy larger than $2.0 \mathrm{MeV}$.

\section{B. Cb-TDHFB equations}

Using the energy functional of Eq. (3), we may derive the $\mathrm{Cb}$-TDHFB equations based on the full TDHFB equation with an assumption that the pair potential is "diagonal" in the canonical basis. Although the Block-Messiah theorem [64] guarantees the existence of the canonical form for the TDHFB state at any time, we need this diagonal assumption of the pair potential in order to keep the Cb-TDHFB equations in a simple form during the time evolution. The details are given in Ref. [29]. The Cb-TDHFB equations are given by

$$
\begin{aligned}
i \frac{\partial}{\partial t}\left|\phi_{k}(t)\right\rangle & =\left(h(t)-\epsilon_{k}(t)\right)\left|\phi_{k}(t)\right\rangle, \\
i \frac{\partial}{\partial t}\left|\phi_{\bar{k}}(t)\right\rangle & =\left(h(t)-\epsilon_{\bar{k}}(t)\right)\left|\phi_{\bar{k}}(t)\right\rangle, \\
i \frac{d}{d t} \rho_{k}(t) & =\kappa_{k}(t) \Delta_{k}^{*}(t)-\kappa_{k}^{*}(t) \Delta_{k}(t), \\
i \frac{d}{d t} \kappa_{k}(t) & =\left(\epsilon_{k}(t)+\epsilon_{\bar{k}}(t)\right) \kappa_{k}(t)+\Delta_{k}(t)\left(2 \rho_{k}(t)-1\right),
\end{aligned}
$$

where the parameters $\epsilon_{k}(t)$ are taken as $\epsilon_{k}(t) \equiv$ $\left\langle\phi_{k}(t)|h(t)| \phi_{k}(t)\right\rangle$, to minimize the phase change of the canonical states. In this article, we adopt the natural unit, 
$\hbar=c=1$. The single-particle Hamiltonian $h(t)$ is defined as usual: a derivative of the energy functional $E[\rho, \kappa]$ with respect to the density, $h_{\mu \nu}(t) \equiv \partial E / \partial \rho_{\nu \mu}$

These are the basic equations to determine the time evolution of the canonical states, $\left|\phi_{k}(t)\right\rangle$ and $\left|\phi_{\bar{k}}(t)\right\rangle$, their occupation $\rho_{k}(t)$, and pair probabilities $\kappa_{k}(t)$. We use the 3D Cartesian coordinate-space representation for the canonical states, $\phi_{k}(\vec{r}, \sigma ; t)=\left\langle\vec{r}, \sigma \mid \phi_{k}(t)\right\rangle$ with $\sigma= \pm 1 / 2$. Thus, each canonical state is represented by three discrete indexes $\left(i_{x}, i_{y}, i_{z}\right)$ for the 3D mesh points; $(x, y, z)=\left(i_{x}, i_{y}, i_{z}\right) \times d$.

\section{Real-time calculation of strength functions}

The $E 1$ strength function in the linear response can be obtained in the real-time calculation with a perturbation of the $E 1$ field. The ground state is prepared by the HF+BCS calculation using the imaginary-time method. Then, we add a weak impulse external field $V_{\text {ext }}(\vec{r}, t)=-\eta \hat{F}_{K}(\vec{r}) \delta(t)$ to start the time evolution. Here, $\hat{F}_{K}(\vec{r})$ is the $E 1$ operator with recoil charges,

$$
\hat{F}_{K}(\vec{r})= \begin{cases}(N e / A) r Y_{1 K}(\hat{r}) & \text { for protons, } \\ -(Z e / A) r Y_{1 K}(\hat{r}) & \text { for neutrons. }\end{cases}
$$

Thus, at time $t=0+$, the initial state of the time evolution is given by $\phi_{k}(\vec{r}, \sigma ; t=0+)=e^{i \eta \hat{F}_{K}(\vec{r})} \phi_{k}^{0}(\vec{r}, \sigma), \rho_{k}(t=0+)=$ $\rho_{k}^{0}$, and $\kappa_{k}(t=0+)=\kappa_{k}^{0}$, where the superscript " 0 " indicates the quantities at the ground state. Then, the expectation value of $\hat{F}$ is calculated as a function of time,

$$
\begin{aligned}
\mathcal{F}_{K}(t) \equiv & \int d \vec{r}\left\{(N e / A) r Y_{1 K}(\hat{r}) \rho_{p}(\vec{r}, t)\right. \\
& \left.-(Z e / A) r Y_{1 K}(\hat{r}) \rho_{n}(\vec{r}, t)\right\},
\end{aligned}
$$

where $\rho_{\tau}(\vec{r}, t)=\sum_{k \in \tau} \rho_{k}(t) \sum_{\sigma}\left|\phi_{k}(\vec{r}, \sigma ; t)\right|^{2}$. The parameter $\eta$ controls the strength of the external field. To calculate the linear response, it should be small enough to validate the linearity. Note that the strength function $S(E ; E 1)$ is independent of magnitude of the parameter $\eta$ as far as the linear approximation is valid. In the present study, we adopt the value of $\eta=3 \times 10^{-5}(\mathrm{e} \mathrm{fm})^{-1}$ for the $E 1$ operator. The time step $d t$ is $0.0005 \mathrm{MeV}^{-1}$ and the time evolution is calculated up to $T=10 \mathrm{MeV}^{-1}$.

The $E 1$ strength function is calculated with the following formula [67]:

$$
\begin{aligned}
S(E ; E 1) & \equiv \sum_{K=-1,0,1} \sum_{n}\left|\left\langle n\left|\hat{F}_{K}\right| 0\right\rangle\right|^{2} \delta\left(E-E_{n}\right) \\
& =-\frac{1}{\pi \eta} \operatorname{Im} \mathcal{F}(E),
\end{aligned}
$$

where $E_{n}$ is the excitation energy of the state $|n\rangle$ and $\mathcal{F}(E)$ is defined by

$$
\mathcal{F}(E)=\sum_{K} \int_{0}^{\infty} w(t) e^{i E t} \mathcal{F}_{K}(t) d t .
$$

Here, we introduce a smoothing function $w(t)$, for which the ideal choice would be unity, $w(t)=1$. However, because of the finite duration of the numerical time evolution, the integration must stop at $t=T$. This requires $w(t)$ to be a function almost vanishing at $t=T$, and leads to the finite energy resolution, $\Delta E \sim 2 \pi T$. In the following calculations, we choose $w(t) \equiv e^{-\Gamma t / 2}$, where $\Gamma$ is a smoothing parameter and is set to $1 \mathrm{MeV}$. This corresponds to the discrete strength smeared with the Lorentzian function with the width of $\Gamma$. For spherical nuclei, different $K$ components in Eq. (11) are all identical. Thus, the actual numerical calculation is performed only for one of the $K$ values, and the final result is obtained by multiplying it by 3 . For axially symmetric nuclei, when the symmetry axis is chosen as the $z$ axis, the $K=1$ and -1 components are identical. The sum of them is simply called " $K=1$ component" in the following.

\section{Definition of PDR fraction}

To quantify the low-lying $E 1$ strength in a systematic investigation, we use the summed energy-weighted $E 1$ strengths in the low-energy region $m_{1}\left(E_{c}\right)$. The ratio of this to the total sum-rule value $m_{1}$,

$$
f_{\mathrm{PDR}}=\frac{m_{1}\left(E_{c}\right)}{m_{1}} \equiv \frac{\int^{E_{\mathrm{c}}} E \times S(E 1 ; E) d E}{\int E \times S(E 1 ; E) d E},
$$

is referred to as "PDR fraction," hereafter. The cutoff energy $E_{c}=10 \mathrm{MeV}$ is adopted in the present calculation. Figure 1 shows the $E 1$ strength function of ${ }^{26} \mathrm{Ne}$ and, the low-lying $E 1$ strength in the current definition is highlighted. The PDR fraction, defined in this way, depends on $E_{c}$ and smoothing parameter $\Gamma$. The present choice of $E_{c}$ can be reasonably justified for relatively light nuclei; however, for heavier deformed isotopes, it is difficult to make a clear separation between the PDR and GDR. Nevertheless, although its absolute magnitude depends on $E_{c}$, the relative quantities are sensible and are useful for discussion of the low-lying $E 1$ strengths.

\section{E. Determination of the model space: Box-size dependence}

The canonical orbits $\phi_{k}(\vec{r}, \sigma)$ are represented in the 3D coordinate space discretized with mesh $d$ in a sphere of radius $R$. It is trivial that the numerical calculation is easier for a smaller model space (smaller $R$ and larger $d$ ). However, the calculation with a too-small model space could produce a

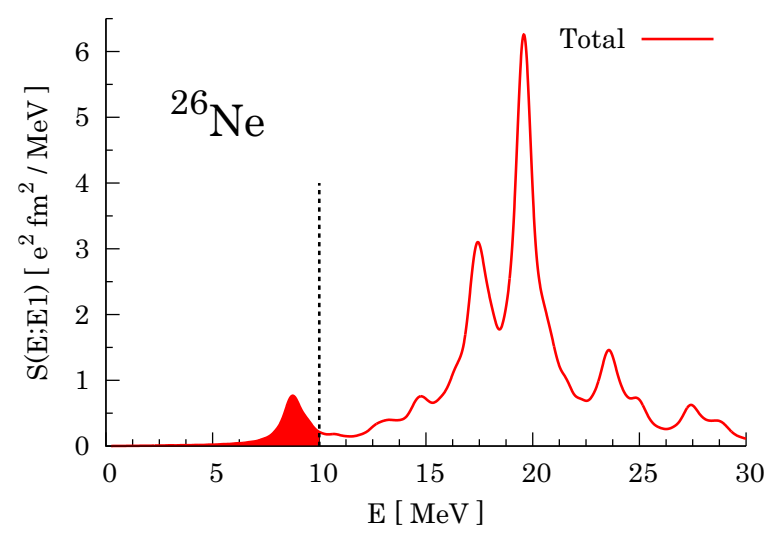

FIG. 1. (Color online) Calculated $E 1$ strength distribution and PDR strength (filled area) for ${ }^{26} \mathrm{Ne}$. A vertical dashed line indicates $E_{c}=10 \mathrm{MeV}$. 


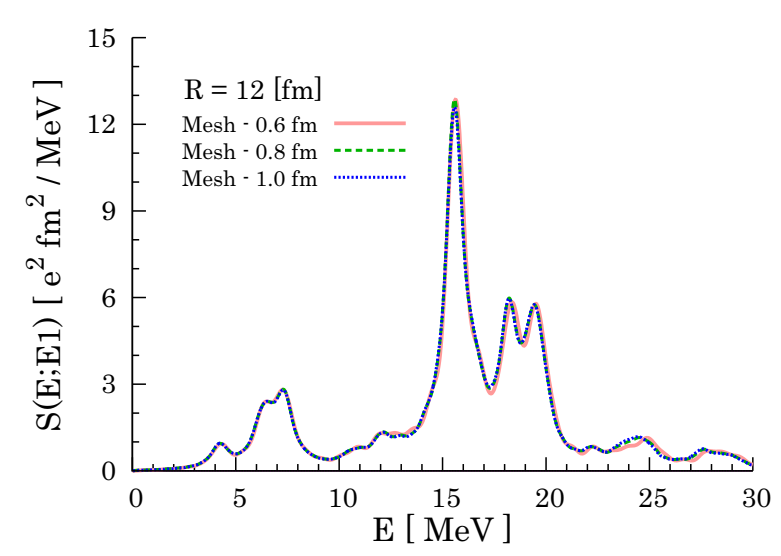

FIG. 2. (Color online) Mesh-size dependence of the strength. These calculations are done in the box of $R=12 \mathrm{fm}$.

qualitatively wrong answer. Roughly speaking, the box size $R$ (mesh size $d$ ) determines the lowest (highest) momentum in the model space. We here show some results to determine these model-space parameters.

Figure 2 shows the mesh-size dependence of the $E 1$ strength function for a very neutron-rich nucleus ${ }^{50} \mathrm{~S}$. The result is well converged with the use of $d \leqslant 1 \mathrm{fm}$. In contrast, the box-size dependence is much more significant, which is illustrated in Fig. 3. The solid, dash-dotted, dashed, and dotted lines correspond to $R=25,20,12$, and $8 \mathrm{fm}$, respectively. In the calculation with the smallest space of $R=8 \mathrm{fm}$, the GDR peak is shifted to higher energy and the low-lying $E 1$ peak disappears. In order to obtain a fully converged result for the $E 1$ strength functions in ${ }^{50} \mathrm{~S}$, we need a very large model space, such as $R \geqslant 30 \mathrm{fm}$. It should be noted that this box-size dependence is significantly weaker in stable nuclei, which was previously investigated in Ref. [51].

It is computationally very demanding to perform a systematic study in such a large model space of $R \geqslant 30 \mathrm{fm}$. Thus, we focus our study on the relative and qualitative behaviors of the low-energy $E 1$ strengths. Figure 4 shows the box-size dependence of the PDR fraction $f_{\mathrm{PDR}}$ for ${ }^{50} \mathrm{~S}$. The result is approximately converged at $R \geqslant 12 \mathrm{fm}$. Figures 5 and 6

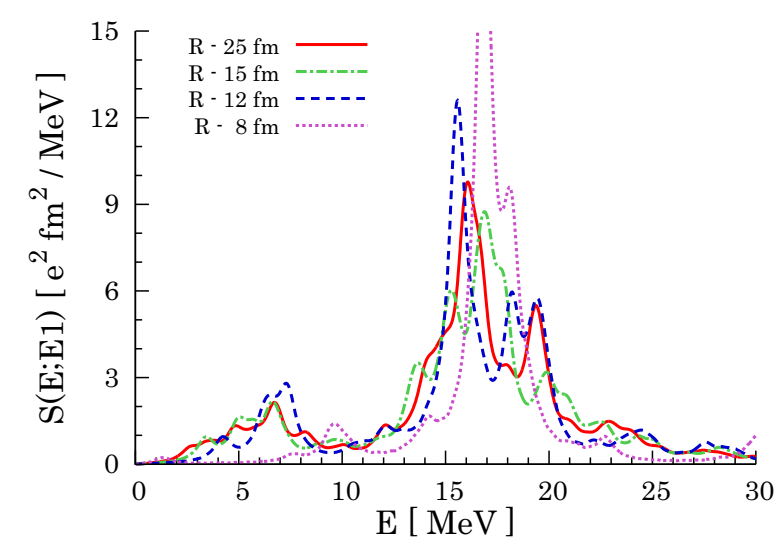

FIG. 3. (Color online) Box-size dependence of the $E 1$ strength for ${ }^{50} \mathrm{~S}$ with mesh size of $d=1 \mathrm{fm}$.

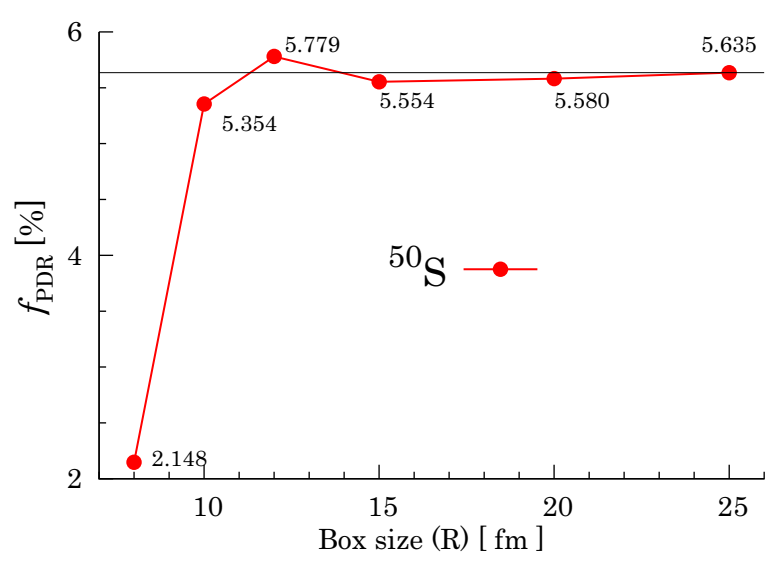

FIG. 4. (Color online) Box-size dependence of the fraction of the low-lying $E 1$ strength for ${ }^{50} \mathrm{~S}$. The thin horizontal line is the result obtained with $R=25 \mathrm{fm}$. The values written around symbols are the fraction ratios obtained in each box.

show the box-size dependence of $S(E 1 ; E)$ and $f_{\mathrm{PDR}}$ for a heavier neutron-rich nucleus, ${ }^{132} \mathrm{Sn}$. The results are almost converged at $R \geqslant 15 \mathrm{fm}$. From these studies, we here adopt the model space as follows: $(R, d)=(12,0.8) \mathrm{fm}$ for isotopes with $Z \leqslant 20$, and $(R, d)=(15,1) \mathrm{fm}$ for heavier isotopes with $28 \leqslant Z \leqslant 50$. These model spaces lead a the number of 3D mesh points of about 8000 .

We should note that the results for extremely neutron-rich nuclei in the present paper may not be fully converged, especially for light isotopes. For instance, we have confirmed that, for Ca isotopes, the $f_{\mathrm{PDR}}$ values calculated with $R=12 \mathrm{fm}$ and $R=15 \mathrm{fm}$ are identical to each other for $N \leqslant 36$, while those for $N>38$ show some difference. However, this difference is about $0.6 \%$ at most, which does not change the conclusion of the present study.

\section{EVOLUTION OF THE LOW-ENERGY E1 STRENGTHS}

In this section, we present how the PDR fraction, Eq. (13), is evolved with respect to neutron number and proton number. All results in this section are obtained with the $\mathrm{SkM}^{*}$ parameter set.

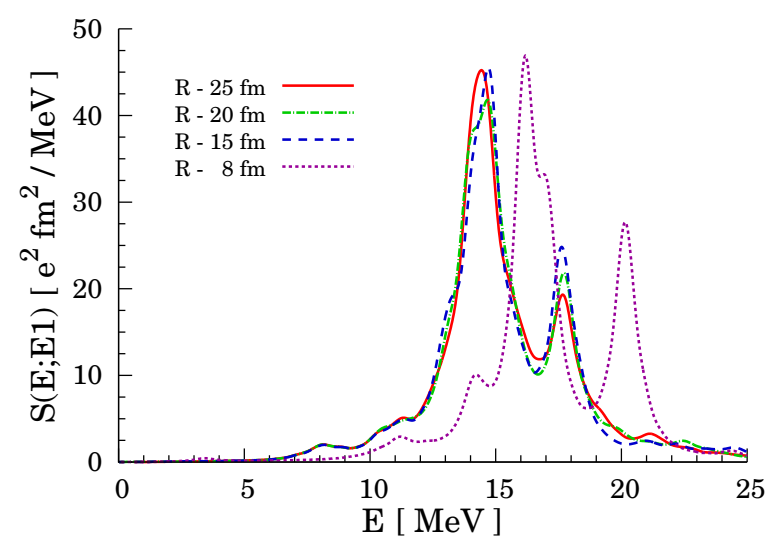

FIG. 5. (Color online) Same as Fig. 3, but for ${ }^{132} \mathrm{Sn}$. 


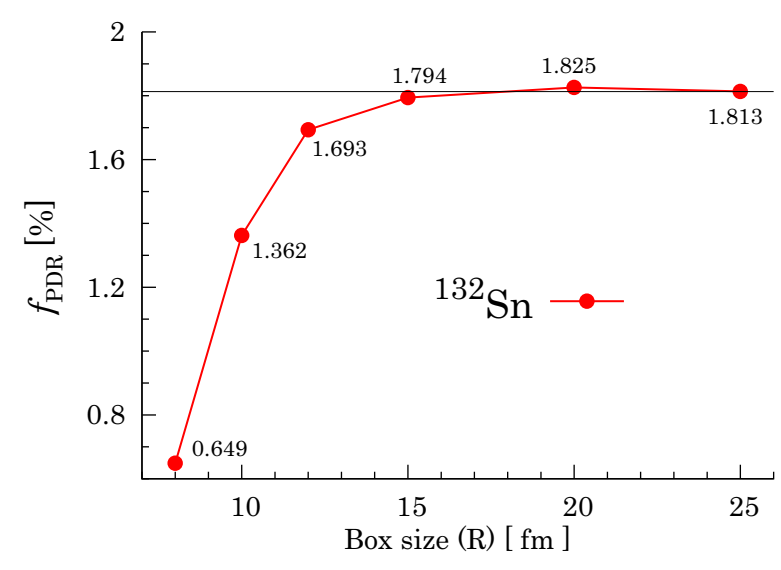

FIG. 6. (Color online) Same as Fig. 4, but for ${ }^{132} \mathrm{Sn}$. The thin line is an eye guide from the result of the $R=25 \mathrm{fm}$ case.

\section{A. Light nuclei in the neutron-rich side $(6 \leqslant Z \leqslant 20$ and $N \geqslant Z)$}

First, we show the behavior of the low-lying $E 1$ strength in relatively light even-even isotopes with $Z=6-20$ which have a chemical potential larger than $2 \mathrm{MeV}$. Table I in the Appendix shows the ground-state properties calculated with the HF+BCS calculation for isotopes with $6 \leqslant Z \leqslant 20$. The results of the HF calculation neglecting the pairing correlation are also shown for comparison. There are some difference in the ground-state deformation between $\mathrm{HF}+\mathrm{BCS}$ and HF calculations, among which ${ }^{32} \mathrm{Mg}$ shows the largest difference, $\beta_{2}^{\mathrm{HF}}=0.35$ and $\beta_{2}^{\mathrm{HF}+\mathrm{BCS}}=0.0$. Nevertheless, for most isotopes, the pairing correlations do not drastically change the ground-state properties.

Figure 7 shows the neutron-number dependence of $f_{\mathrm{PDR}}$ for $\mathrm{C}, \mathrm{O}, \mathrm{Ne}$, and $\mathrm{Mg}$ isotopes. The solid lines with filled symbols indicate the present results, which can be compared with those of the Hartree-Fock plus random-phase approximation (HF+RPA) [57] presented by the dashed lines with open

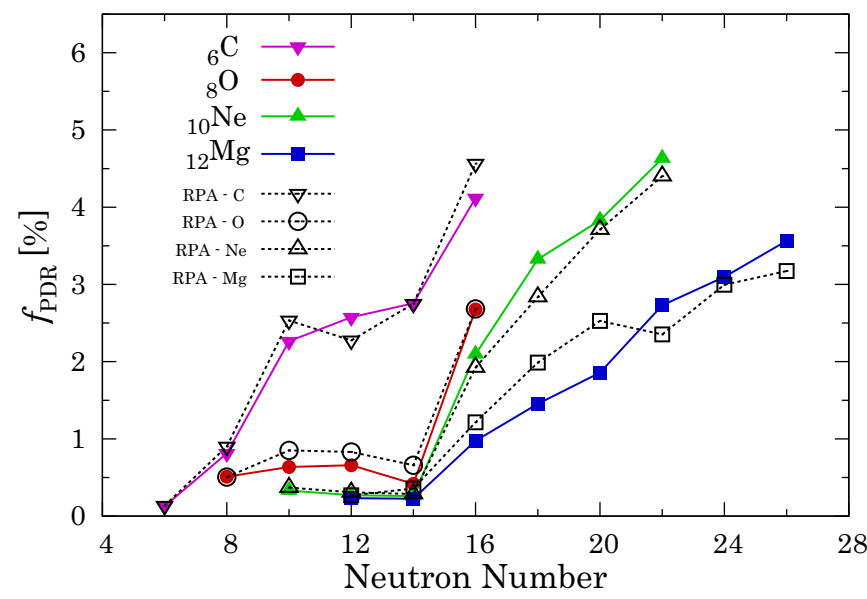

FIG. 7. (Color online) Fraction of the low-lying $E 1$ strength $f_{\text {PDR }}$ in Eq. (13) as functions of the neutron number for $\mathrm{C}, \mathrm{O}, \mathrm{Ne}$ and $\mathrm{Mg}$ isotopes. The solid lines with filled symbols show the present results of Cb-TDHFB, while the dashed lines with open symbols show those of $\mathrm{HF}+\mathrm{RPA}$. symbols. The two kinds of calculations produce qualitatively the same results. This confirms that the pairing plays a minor role in the low-energy $E 1$ strength function for these light nuclei.

The isotopes with $Z=8-12$ have $f_{\mathrm{PDR}}$ less than $1.0 \%$ for $N \leqslant 14$. Then, there is a sudden jump in $f_{\mathrm{PDR}}$ at $N=$ $14 \rightarrow 16$ on every isotopic chain. The neutron number $N=16$ corresponds to the occupation of $s_{1 / 2}$ orbit. The important role of the weakly bound $s_{1 / 2}$ orbit in the low-energy $E 1$ strengths has been discussed in Ref. [57]. The present result confirms that the pairing correlations do not change the main conclusion. Note that the neutrons are in the normal phase $\left(\Delta_{n}=0\right)$ for nuclei with $N=16$. The largest deviation from the HF+RPA result is seen in ${ }^{32} \mathrm{Mg}$, which is due to the large difference in the ground-state deformation.

The HF+RPA calculation predicts that the next jump in $f_{\mathrm{PDR}}$ is at $N=28 \rightarrow 30$ [57]. $N=30$ correspondS to the occupation of $p_{3 / 2}$ orbit. This is shown in Fig. 8, for $\mathrm{S}, \mathrm{Ar}$, and $\mathrm{Ca}$ isotopes. The qualitative behaviors are identical to those of the HF+RPA calculation. For $\mathrm{Si}$, the kink of $f_{\mathrm{PDR}}$ disappears because the $N=28$ magicity becomes weak in the neutron-rich $\mathrm{Si}$ isotopes, leading to deformed shapes in the mean-field calculation (see Table I). However, for $\mathrm{S}$ and Ar isotopes, the sudden jump at $N=28 \rightarrow 30$ predicted by the HF+RPA is now replaced by a gradual increase in the slope around $N=28$. This smooth evolution of the $f_{\mathrm{PDR}}$ is caused by the fractional occupation probability of the special single-particle states, such as $p_{3 / 2}$ and $p_{1 / 2}$ orbits, due to the pairing correlation. Again, the occupation of weakly bound orbits with low orbital angular momenta $($ low- $\ell$ ) increases the low-energy $E 1$ strength. Beyond $N=34$, the neutrons start occupying the $f_{5 / 2}$ orbit, which reduces the slope in $f_{\mathrm{PDR}}$.

In contrast, for $\mathrm{Ca}$ isotopes, the sudden jump at $N=$ $28 \rightarrow 30$ survives in the present calculation, mainly because of a large shell gap at $N=28$ which makes the neutron pairing gap vanish $\left(\Delta_{n}=0\right)$. At $N=34, f_{\mathrm{PDR}}$ in the present calculation becomes smaller than the result from HF+RPA. This is due to the pairing effect. In the HF calculation, the ground state in ${ }^{54} \mathrm{Ca}$ corresponds to the full occupation of the neutron $p_{1 / 2}$ orbit. However, the $\mathrm{HF}+\mathrm{BCS}$ calculation

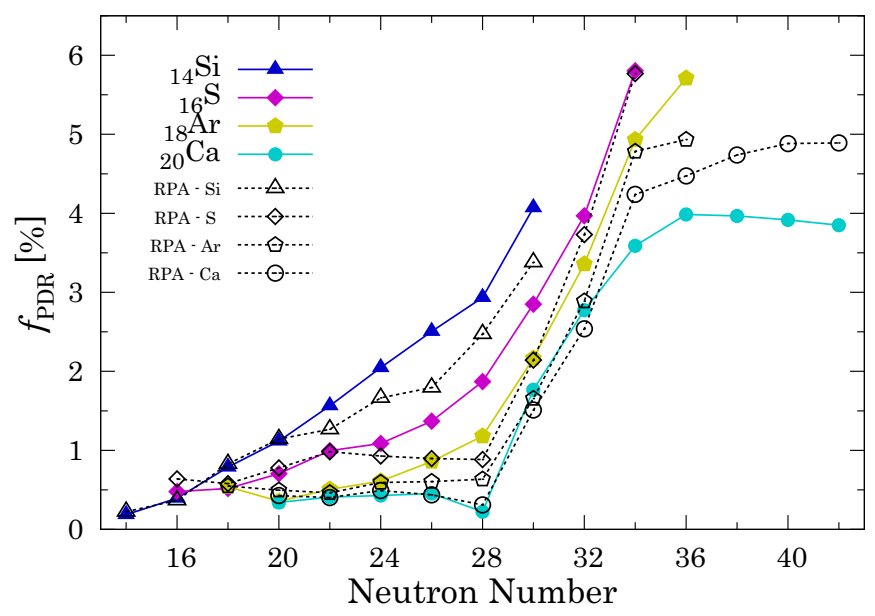

FIG. 8. (Color online) Same as Fig. 7, but for $\mathrm{Si}, \mathrm{S}, \mathrm{Ar}$ and $\mathrm{Ca}$ isotopes. 
produces fractional occupation probabilities of $p_{1 / 2}(52.4 \%)$ and $f_{5 / 2}$ orbits $(18.2 \%)$.

\section{B. Medium-heavy nuclei in the neutron-rich side $(\mathbf{2 8} \leqslant \boldsymbol{Z} \leqslant \mathbf{5 0}$ and $N \geqslant \boldsymbol{Z})$}

In Fig. 9, we show the neutron number dependence of $f_{\mathrm{PDR}}$ for isotopes with $Z=28-50$. The characteristic cusps can be seen at $N=50$ and 82 , which correspond to the neutron magic numbers. While the neutrons are filling the $g_{9 / 2}$ intruder orbit $(40<Z \leqslant 50)$, the PDR fraction stays approximately constant with respect to the neutron number. Especially, the isotopes with $Z=36-50(\mathrm{Kr}-\mathrm{Sn})$ have roughly identical values of $f_{\mathrm{PDR}}$ at $N \leqslant 50$. Beyond $N=50$, the neutrons start occupying the $d_{5 / 2}$ orbit; then, the rapid increase in $f_{\mathrm{PDR}}$ is clearly observed in Fig. 9. These are universal for all the isotopes shown in Fig. 9, although the cusp behavior is weakened by increasing the proton number. These are similar to the cusps at $N=14$ and 28 in lighter neutron-rich isotopes.

In addition, for the isotopes with $Z=32-44$, the convex cusps also appear at around $N=58-60$ and around $N=$ $72-74$, while the concave ones can be seen at $N=60-62$. These cusps are most prominent around the proton subshell $(Z \approx 40$ ), while they become weaker approaching the magic numbers, $Z \rightarrow 28$ and $Z \rightarrow 50$. This suggests that these may be associated with the ground-state deformation.

Let us briefly comment on the deformation effect. In $\mathrm{Ne}$ and $\mathrm{Mg}$ neutron-rich isotopes, the present results suggest that the onset of deformation in the ground state increases $f_{\mathrm{PDR}}$. However, the behavior in the present mass region is more complex. $f_{\mathrm{PDR}}$ decrease at the onset of deformation around; then, they decrease again near $N=74$, which corresponds to the disappearance of the deformation back to the spherical shape. In Sec. IV, we discuss the effect of deformation in more details.

The next jump in $f_{\mathrm{PDR}}$ at $N=82 \rightarrow 84$ is clearly identified. This suggests that the definition of the "low- $\ell$ orbits" is different between light and heavy systems. The nuclei around $N=82$ are all calculated to be spherical, thus it cannot be the effect of deformation. The single-particle orbit just above the $N=82$ shell gap is $f_{7 / 2}$. In light nuclei, when the Fermi level

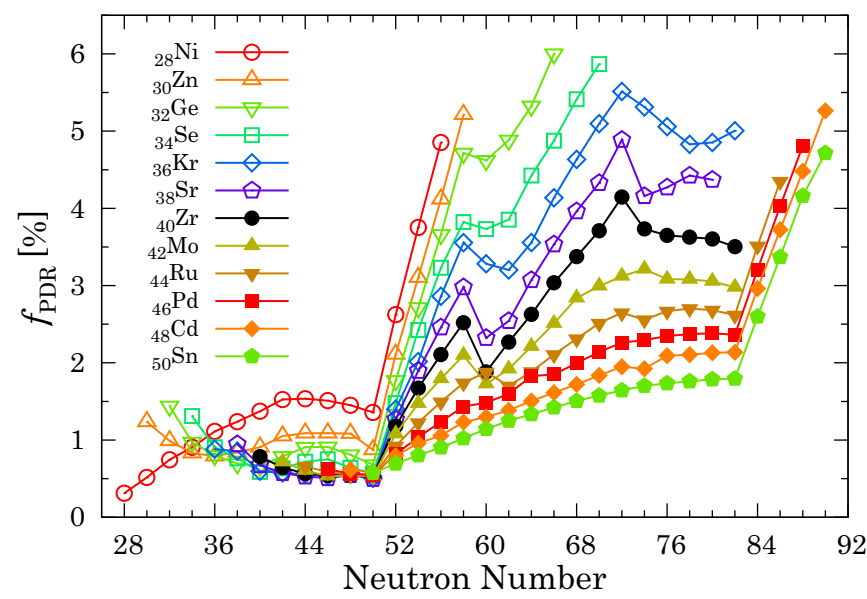

FIG. 9. (Color online) The PDR fraction $f_{\mathrm{PDR}}$ as functions of the neutron number for even-even isotopes with $Z=28-50$ and $N \geqslant Z$. is located at the $f_{7 / 2}$ intruder orbits $(20<N \leqslant 28)$, the PDR fraction does not increase (see Fig. 8). The behavior of $f_{\mathrm{PDR}}$ in the heavy isotopes seems to be very different from that in light systems. The $f$ orbit may be regarded as the low- $\ell$ orbit for heavy nuclei with $N>82$.

Finally, the effect of pairing should be noted. In Ref. [57], similar studies with the HF+RPA were reported for isotopes with $Z \leqslant 40$. The neutron shell effect on $f_{\mathrm{PDR}}$ is qualitatively identical. However, the HF calculation for heavy isotopes shows peculiar changes in the ground-state deformation from one nucleus to the next, which leads to irregular behaviors in $f_{\mathrm{PDR}}$ in the region of $N>56$. These irregular behaviors in Ref. [57] are hindered in the present study. This is due to the pairing correlation, which produces the fractional occupation probabilities, suppressing the sudden changes in deformation from nucleus to nucleus. Now, some systematic trends in the region $56<N<82$ can be observed in Fig. 9. For instance, increasing the proton number from $Z=28$, the kink behavior around $N=60$ becomes sharper toward $Z=38(\mathrm{Sr})$ and $40(\mathrm{Zr})$; then, beyond $Z=40$, it becomes weaker and disappears near $Z=50$ ( $\mathrm{Cd}$ and $\mathrm{Sn})$. A similar systematic behavior can be also observed for kinks around $N=72$.

\section{Neutron skin thickness and PDR fraction}

The classical picture of the PDR is a vibration of neutronskin against the core part, from which, the correlation between skin-thickness and PDR is expected. In this section, we perform a systematic investigation and present the correlation between $f_{\mathrm{PDR}}$ and the skin thickness $\Delta r_{\text {rms }}$ for many isotopes. The neutron skin thickness is defined by the difference in root-mean-square radius of neutrons and protons, $\Delta r_{\text {rms }} \equiv$ $\sqrt{\left\langle r^{2}\right\rangle_{n}}-\sqrt{\left\langle r^{2}\right\rangle_{p}}$.

Figure 10 shows $f_{\mathrm{PDR}}$ as a function of the neutron skin thickness. For $\mathrm{Ge}, \mathrm{Se}, \mathrm{Kr}, \mathrm{Sr}$, and $\mathrm{Zr}$ isotopes, a similar investigation was performed with the HF+RPA [57]. A consistent behavior with Ref. [57] is confirmed in the left panel of Fig. 10. There is a linear correlation between $f_{\mathrm{PDR}}$ and

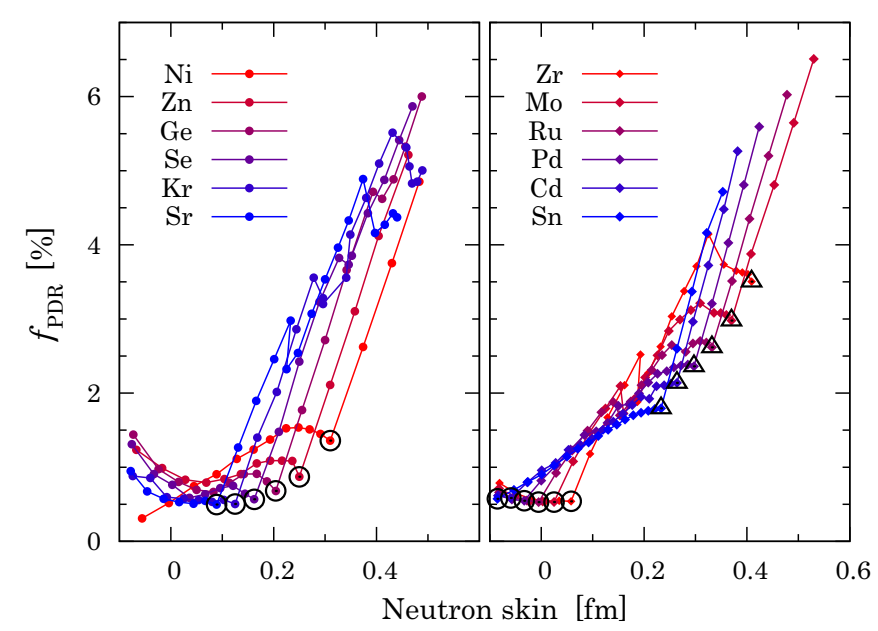

FIG. 10. (Color online) The PDR fractions as functions of the neutron skin thickness for even-even isotopes with $Z=28-50$. The open circles (triangles) indicate those with $N=50$ (82). 
$\Delta r_{\text {rms }}$ for $N=50-58$ with a roughly identical slope. In fact, the slope, $d f_{\mathrm{PDR}} / d \Delta r_{\mathrm{rms}}$, gradually decreases as the proton number increases. This can be clearly seen in the right panel of Fig. 10 for isotopes with $Z=40-50$. As the proton number increases, the neutron Fermi level is more deeply bound, and the slope at $N=50-58$ decreases from Mo to $\mathrm{Sn}$. The slope $d f_{\mathrm{PDR}} / d \Delta r_{\mathrm{rms}}$ in Sn isotopes is roughly half of those shown in the left panel of Fig. 10. Beyond $N=82, d f_{\mathrm{PDR}} / d \Delta r_{\text {rms }}$ again increase and stay almost constant (a linear correlation). The observed slopes at $N>82$ are almost identical to those in the left panel with $Z=28-38$ at $N=50-58$.

The properties of the correlation between the PDR strength and the neutron skin thickness turn out to be rather complex, depending on both the proton and the neutron numbers. Nevertheless, in the present calculation, we observe a universal behavior which is the maximum gradient for $\mathrm{Ni}$ isotopes, $\left(d f_{\mathrm{PDR}} / d \Delta r_{\mathrm{rms}}\right)_{\max } \approx 0.26 \mathrm{fm}^{-1}$. The maximum values appears at $N>50$ and $N>82$ in which the neutron Fermi levels are located at weakly bound $d_{5 / 2}$ and $f_{7 / 2}$ orbits, respectively. It should be noted that a similar maximum value of $\left(d f_{\mathrm{PDR}} / d \Delta r_{\mathrm{rms}}\right)_{\max } \approx 0.2 \mathrm{fm}^{-1}$ was previously observed in the HF+RPA calculation in the lighter mass region at $N>28$, in which the neutrons are filling weakly bound $p$ orbits [57].

\section{Analysis with the SkI3 energy functional}

The PDR properties predicted by the relativistic mean-field (RMF) theories are often quantitatively different from those obtained with the nonrelativistic Skyrme energy functionals, although they show qualitative agreements [68]. For instance, in the present calculation with the $\mathrm{SkM}^{*}$ functional, the PDR strength $f_{\mathrm{PDR}}$ for ${ }^{132} \mathrm{Sn}$ is less than $2 \%$, while it is more than $4 \%$ in the relativistic calculation with the DD-ME2 [68], FSUGold, and NL3 [34] functionals. The experimental data suggested the value of about 4\% [12]. To investigate the origin of the difference, we adopt the SkI3 Skyrme parameter set which has the same density dependence of the spin-orbit form factor as that of the RMF [69].

Figure 11(a) shows the neutron number dependence of $f_{\mathrm{PDR}}$ in $\mathrm{Sn}$ isotopes, which are obtained by $\mathrm{Cb}$-TDHFB calculation with $\mathrm{SkM}^{*}$ (filled circle) and SkI3 (filled square). We can see a clear difference between them. The result of SkI3 shows rapid increase of $f_{\mathrm{PDR}}$ as a function of neutron number in the regions of $58<N<70$ and $N>82$. It shows constant values of $f_{\mathrm{PDR}} \approx 4 \%$ in $70 \leqslant N \leqslant 82$, corresponding to the neutron Fermi level in the intruder $h_{11 / 2}$ orbit. These behaviors produce prominent kinks at $N=70$ and 82 in Fig. 11(a). This is consistent with the RMF results of Refs. [34,68].

The single-particle levels of ${ }^{134} \mathrm{Sn}$ obtained with the SkM* and the SkI3 parameter sets are shown in Fig. 11(c). The level spacings in the region of $50<N<82$ are much larger in SkI3 than in $\mathrm{SkM}^{*}$. Note that the effective mass $\left(\mathrm{m}^{*} / \mathrm{m}\right)$ in $\mathrm{SkI} 3$ is 0.58 which is significantly smaller than 0.79 in $\mathrm{SkM}^{*}$. In addition, the level orderings of $g_{7 / 2}$ and $d_{5 / 2}$ are different. These properties lead to a prominent subshell effect on the PDR with SkI3. Namely, after the full occupation of the $g_{7 / 2}$ orbit at $N=58$, while the neutrons start to fill the low- $\ell$ $\left(d\right.$ and $s$ ) orbits, $f_{\mathrm{PDR}}$ shows a rapid increase. Then, beyond $N=70, f_{\text {PDR }}$ stops increasing, since the neutrons now fill the intruder orbit $h_{11 / 2}$. These effects are much weaker in SkM* because the single-particle levels are much denser and the subshell effects are smeared out.

In Fig. 11(b), we show $f_{\mathrm{PDR}}$ as a function of the neutron skin thickness. It shows a clear discrepancy between the results with $\mathrm{SkM}^{*}$ and $\mathrm{SkI} 3$, which is associated with the different subshell effects. Apparently, the PDR strength is not uniquely determined by the neutron skin thickness, but is affected by the choice of the energy functional and the associated single-particle structure. In contrast, the rapid increase of $f_{\mathrm{PDR}}$ at $N>82$ is a universal feature. The slopes $d f_{\mathrm{PDR}} / d \Delta r_{\mathrm{rms}}$ are also similar to each other, although the slope in SkI3, $d f_{\mathrm{PDR}} / d \Delta r_{\mathrm{rms}} \approx 0.37 \mathrm{fm}^{-1}$, is slightly steeper than that in $\mathrm{SkM}^{*}$.

In summary, the PDR strength significantly depends on the single-particle shell structure, and thus has a functional dependence. In fact, even in the relativistic calculations, the ones adopting the point-coupling energy functionals show results very similar to the present calculation with $\mathrm{SkM}^{*}$ [40]. This strong shell effect of the PDR may be useful for investigating the neutron single-particle levels in unstable nuclei.
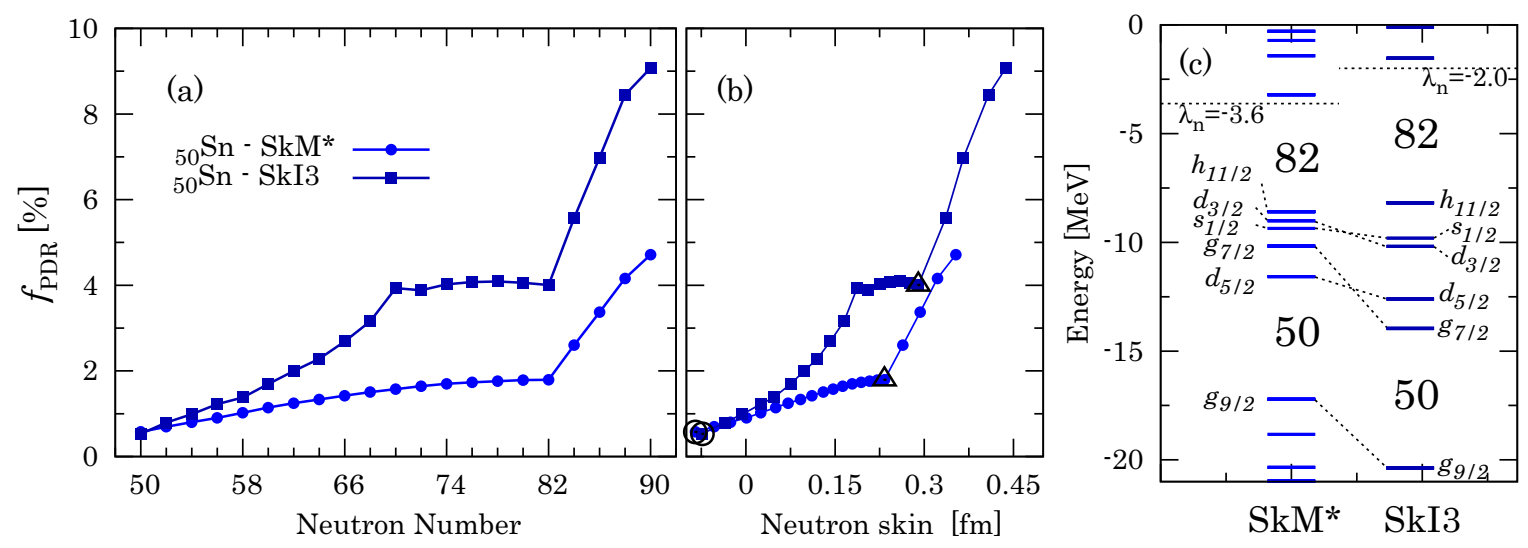

FIG. 11. (Color online) (a) The PDR fraction of Sn isotopes as a function of neutron number, calculated with the SkM* energy functional (circles) and with SkI3 (squares). (b) The same as (a) but as a function of the neutron skin thickness. Open circles (triangles) indicate $N=50$ (82). (c) Neutron single-particle levels around chemical potential $\lambda_{n}$ for ${ }^{134} \mathrm{Sn}$, calculated with SkM* and SkI3 functionals. 


\section{DEFORMATION EFFECTS}

It is well known that the nuclear deformation in the ground state significantly affects the shape of the GDR peak [70-73]. For a well deformed nucleus, the GDR peak is split into two peaks, which can be understood as a simple geometrical effect. Namely, there are different frequencies associated with long and short principal axes of deformation. On the other hand, for the low-lying $E 1$ mode, the geometrical effect is not prominent. The authors of Ref. [74] have reported that the deformation hinders the PDR strength for the prolate neutronrich $\mathrm{Sn}$ isotopes. They also found that the $K=0$ component of the calculated low-lying $E 1$ strength is larger than the $K=1$ component, which was interpreted by the effect of anisotropic neutron skins. Namely, the neutron skin thickness in the $z(K=0)$ direction is expected to be thicker than in the $x-y(K=1)$ directions. In this section, we investigate this deformation effect for selected isotopes: strongly deformed ( $\mathrm{Sr}$ and $\mathrm{Zr}$ ) and weakly deformed nuclei (Pd and $\mathrm{Cd}$ ).

\section{A. Deformation and PDR strength}

In Fig. 12, $f_{\mathrm{PDR}}$ are shown for $\mathrm{Zr}$ isotopes. In the present calculation, $\mathrm{Zr}$ isotopes with $N=60-72$ are deformed with a prolate shape, and the one with $N=74$ has a small triaxial shape. The other nuclei are calculated to be spherical in the ground state. Here, we can clearly identify the deformation effects. The total PDR strength suddenly reduces at $N=58 \rightarrow$ 60 at the onset of deformation $\left(\beta_{2} \approx 0.37\right)$. This is consistent with Ref. [74], in that the deformation hinders the PDR strength. However, at $N=72 \rightarrow 76$ where the deformation reduces and disappears, the PDR strength decreases again. Thus, the deformation does not always hinder the PDR strength.

Very similar behaviors can be found for $\mathrm{Sr}$ isotopes as well, in Fig. 13. In $\mathrm{Sr}$ isotopes, the ground state has a prolate shape with $\beta_{2} \approx 0.37$ for $N=60-72$, and an oblate shape at $N=38$ and 74 . When the prolate deformation develops in the ground

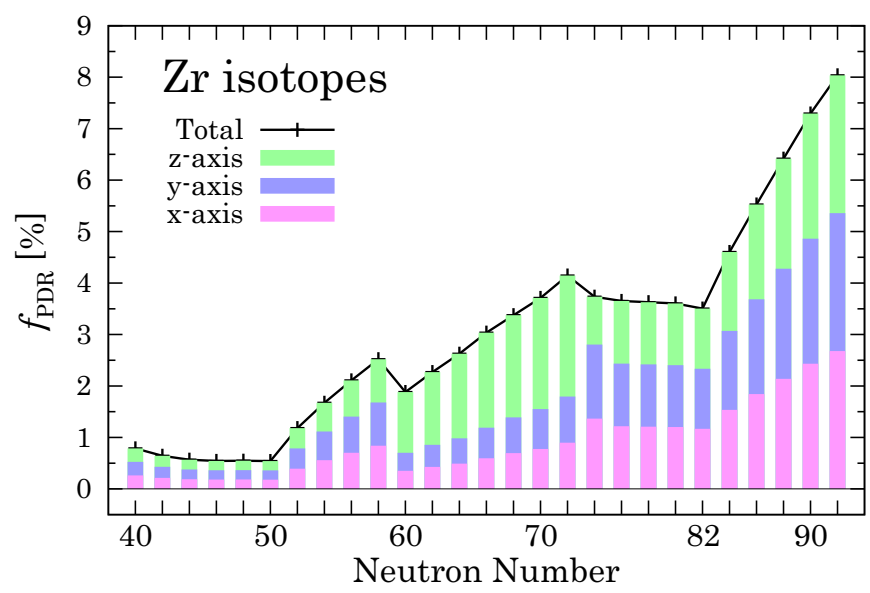

FIG. 12. (Color online) The PDR strength in Zr isotopes decomposed into $x, y$, and $z$ components which are denoted by red, blue, and green bars. In spherical nuclei, the three components are equal to each other. In this figure, we include isotopes with the neutron chemical potentials smaller than $2 \mathrm{MeV}(N>82)$.

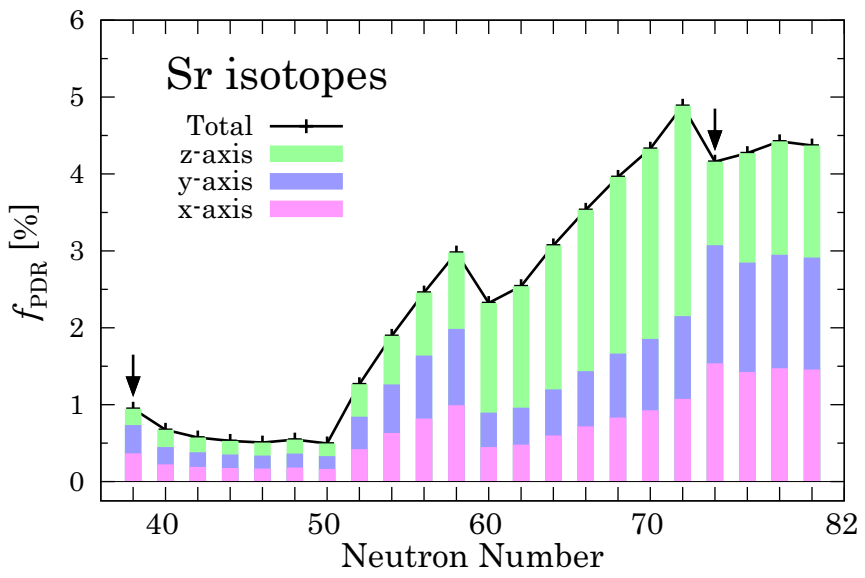

FIG. 13. (Color online) Same as Fig. 12, but for Sr isotopes. The arrows indicate the oblate-shape nuclei.

state, the PDR strength reduces. The largest PDR strength is seen in $N=72$, then it drops at $N>72$ as the ground state is going back to the spherical shape.

In weakly deformed isotopes, the deformation effect on the PDR is much milder. In Fig. 14, we show Pd isotopes. The ground state of $\mathrm{Pd}$ isotopes is deformed with a prolate shape at $N=58-74$; however, their deformation $\beta_{2}=0.1-0.2$ is significantly smaller than that of $\mathrm{Zr}$ isotopes. The PDR strength gradually increases toward $N=82$, then jumps up beyond that. In $\mathrm{Cd}$ isotopes, we observe behaviors almost identical to $\mathrm{Pd}$.

We have found that the isotopic dependence of the PDR strength is consistent with the behavior of the neutron chemical potentials. In Figs. 15 and 16, the neutron chemical potentials $-\lambda_{n}$ are shown for $\mathrm{Zr}$ and $\mathrm{Pd}$ isotopes, respectively. In $\mathrm{Zr}$ isotopes, the sudden change of the deformation, in fact, causes the change in $\lambda_{n}$ as well. The chemical potential $\left|\lambda_{n}\right|$ monotonically decreases with increasing neutron number, except for two places. These exceptional increases of $\left|\lambda_{n}\right|$ take place at $N=58 \rightarrow 60$ and at $N=72 \rightarrow 76$. These neutron numbers perfectly agree with those showing the drops of the PDR strength in neutron-rich $\mathrm{Zr}$ isotopes. In contrast, the

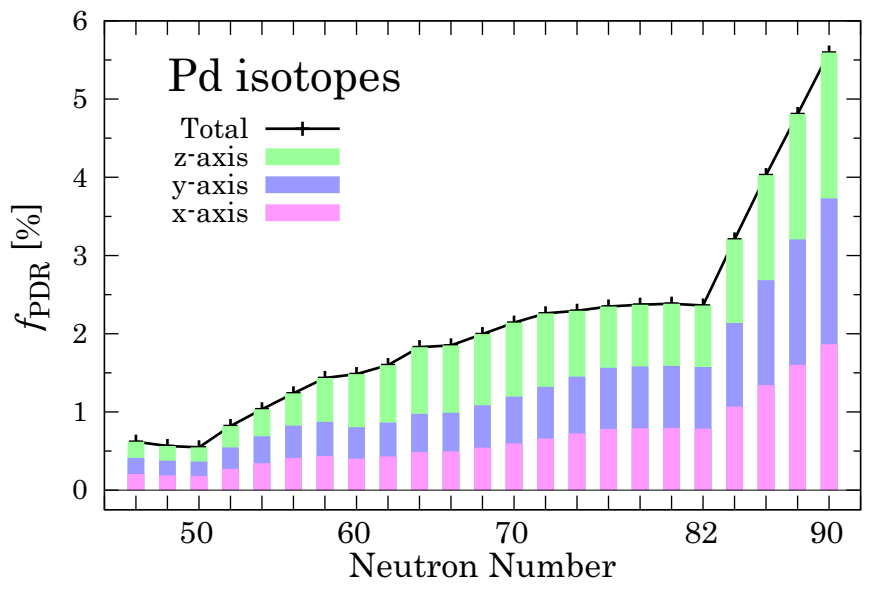

FIG. 14. (Color online) Same as Fig. 12, but for Pd isotopes. 


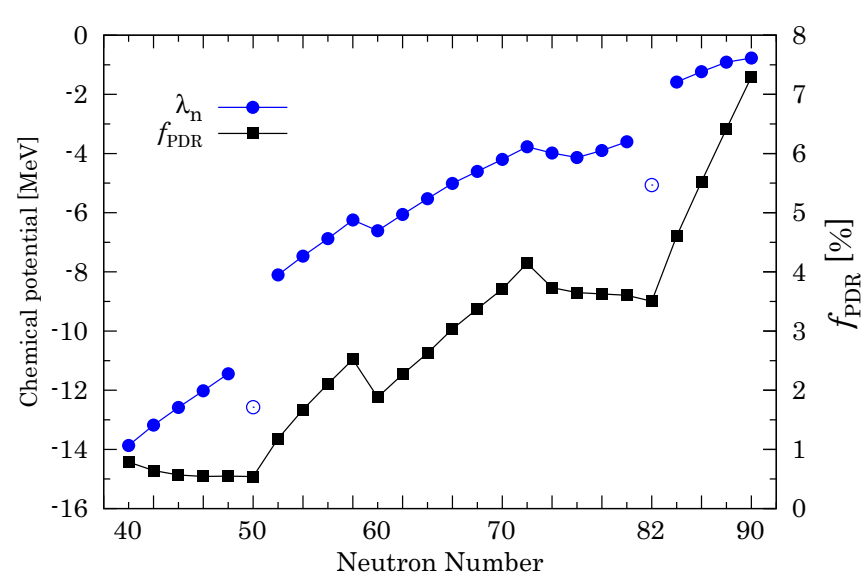

FIG. 15. (Color online) Neutron chemical potentials for $\mathrm{Zr}$ isotopes as a function of neutron number are shown by circles. At $N=50$ and 82 , the neutron pairing gaps vanish, for which the highest-occupied single-particle levels are shown by open circles. The PDR fraction $f_{\mathrm{PDR}}$ are also shown by squares, and their scale is given in the right axis.

$\left|\lambda_{n}\right|$ in $\mathrm{Pd}$ isotopes show a monotonic decrease, leading to a monotonic increase in $f_{\mathrm{PDR}}$. Therefore, it seems that the deformation affects the PDR strength through changing the neutron chemical potential.

\section{B. The $K=0$ dominance in PDR strength}

In GDR at high frequency $E 1$ mode, the deformation splitting was systematically observed in deformed nuclei. The $K=0$ component is lower in energy than the $K=1$ component, leading to a double-peak structure in the prolate nuclei. In oblate nuclei, the opposite situation is expected, so that the $K=0$ is higher than $K=1$. In Figs. 12, 13, and 14, the PDR strengths are decomposed into $x, y$, and $z$ components. Here, the $z$ direction is chosen as the symmetry axis of the deformation. The calculation indicates that, in the prolate nuclei, the $K=0(z)$ PDR components are larger than the $K=1$ components. This is consistent with Ref. [74], in which it was interpreted to be due to the difference in the neutron skin thickness with respect to the $z$ and $x-y$ directions. In

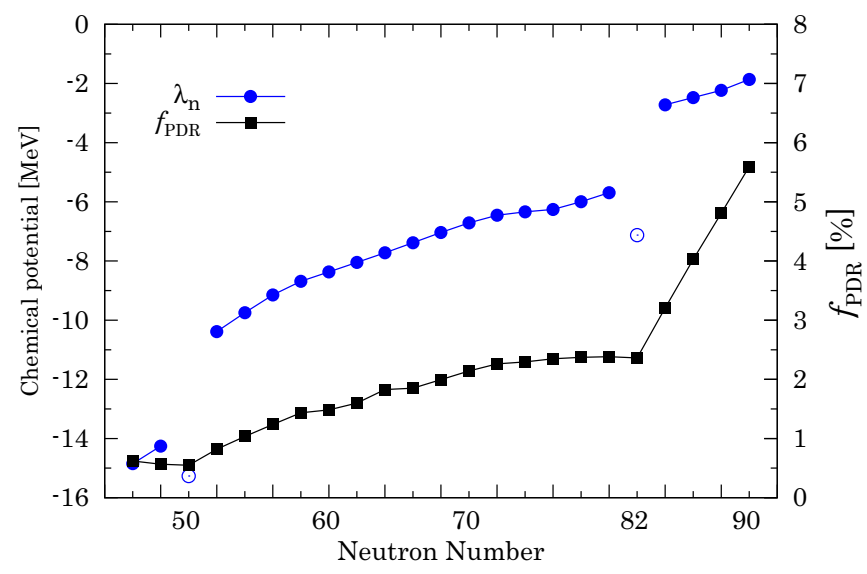

FIG. 16. (Color online) Same as Fig. 15, but for Pd isotopes.

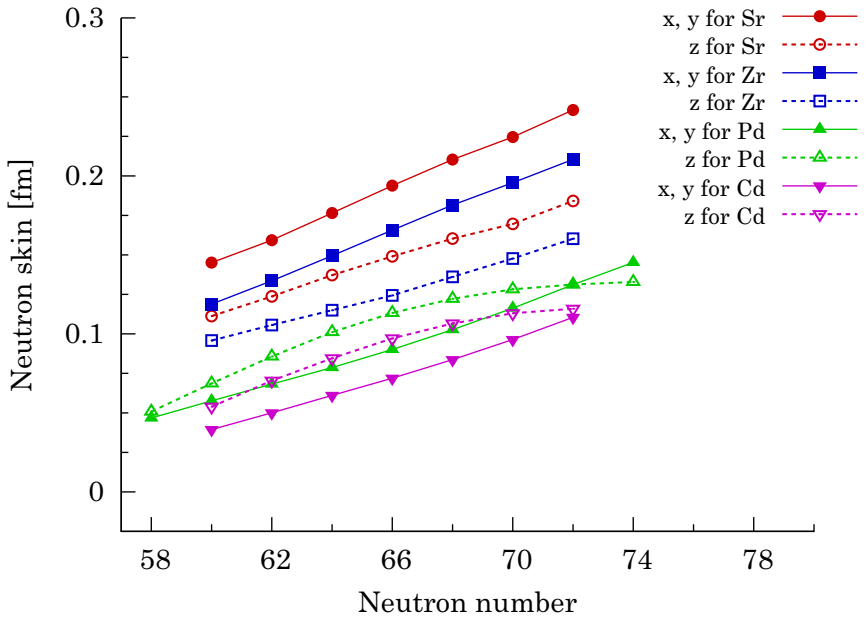

FIG. 17. (Color online) Neutron skin thickness in different directions for prolate deformed $\mathrm{Sr}, \mathrm{Zr}, \mathrm{Pd}$, and $\mathrm{Cd}$ isotopes. The values along $z$ and the perpendicular directions are shown by empty and filled symbols, respectively.

Ref. [74], for very neutron-rich Sn isotopes with $N=92-112$, the prolate deformation of the neutron density was calculated to be larger than that of protons $\left[\left(\beta_{2}\right)_{n}>\left(\beta_{2}\right)_{p}\right]$. This leads to a larger neutron skin in the $z$ direction of the symmetry axis than in the perpendicular directions $(x-y)$. However, it turns out that, in the present calculation, the situation in $\mathrm{Sr}$ and $\mathrm{Zr}$ isotopes is very different from $\mathrm{Sn}$ isotopes in Ref. [74].

Figure 17 shows the neutron skin thickness with respect to $z$ and $x-y$ directions for prolate deformed $\mathrm{Sr}, \mathrm{Zr}, \mathrm{Pd}$, and $\mathrm{Cd}$ isotopes. These are defined by the root-mean-square radius of the $z$ direction, $\sqrt{\left\langle z^{2}\right\rangle_{n}}-\sqrt{\left\langle z^{2}\right\rangle_{p}}$, shown by dashed lines, and the same for the $x$ and $y$ directions shown by solid lines. It turns out that in $\mathrm{Sr}$ and $\mathrm{Zr}$ isotopes, the neutron skin is thicker in the $x-y$ plane than in the $z$ direction, while the situation is opposite in $\mathrm{Pd}$ and $\mathrm{Cd}$ isotopes.

In Fig. 18, we show the calculated quadrupole deformation $\beta_{2}$ for protons and neutrons separately. In $\mathrm{Pd}$ and $\mathrm{Cd}$ isotopes,

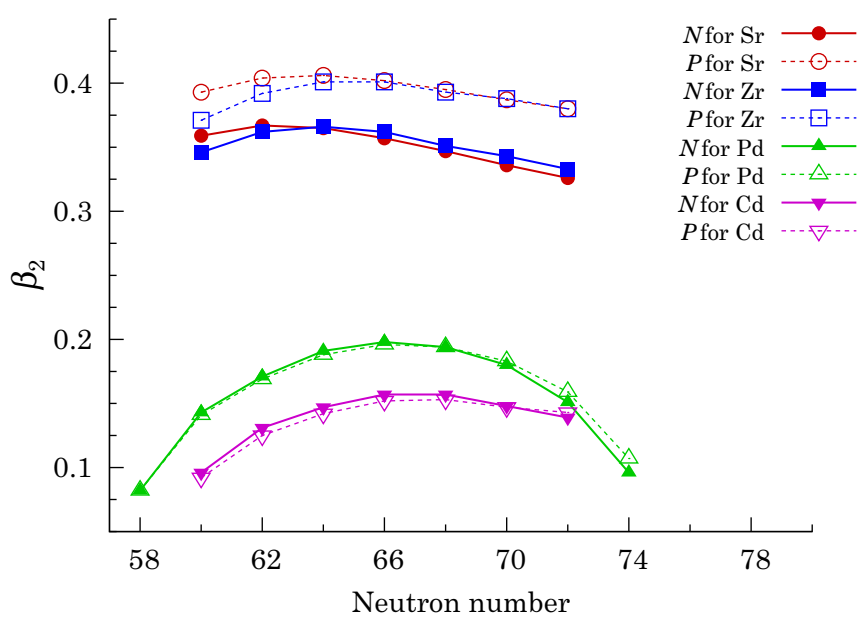

FIG. 18. (Color online) Neutron and proton quadrupole deformations, $\left(\beta_{2}\right)_{n}$ and $\left(\beta_{2}\right)_{p}$, are respectively shown by filled and open symbols, for prolate deformed $\mathrm{Sr}, \mathrm{Zr}, \mathrm{Pd}$, and $\mathrm{Cd}$ isotopes. 


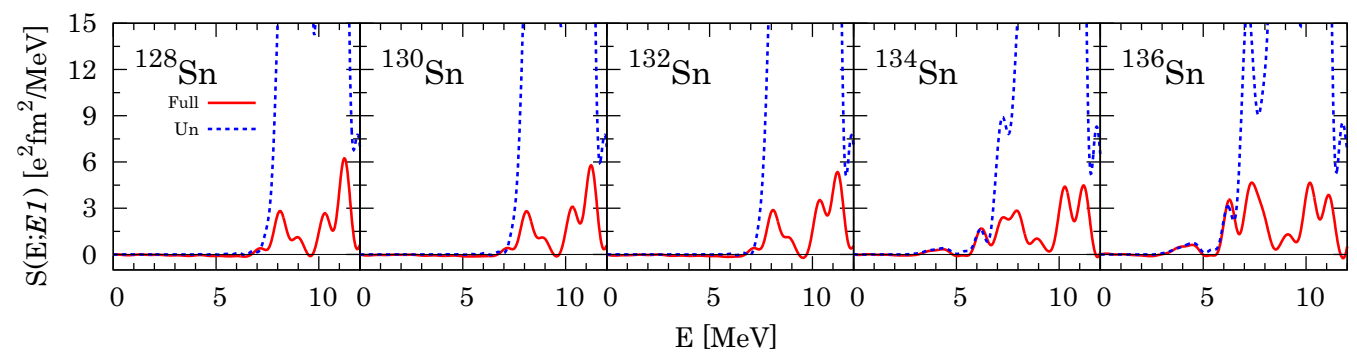

FIG. 19. (Color online) $E 1$ strength functions below $12 \mathrm{MeV}$ for $\mathrm{Sn}$ isotopes with $N=78-86$, calculated with the SkM* parameter set. The solid and dashed lines show full and unperturbed strength distributions. For the function $w(t)$, Eq. (15) is used.

the deformation of the proton density distribution is identical to that of neutrons. However, for $\mathrm{Sr}$ and $\mathrm{Zr}$ isotopes, the deformation is actually larger for protons than for neutrons. Thus, the skin thickness enhances in the perpendicular directions $(x-y)$ as is shown in Fig. 17. If the neutron skin thickness is directly related to the PDR strength, we could expect the $K=1$ dominance, rather than the $K=0$ dominance. This is opposite to what we have observed in the present calculation (Figs. 12 and 13). The anisotropy of the neutron skin thickness is unlikely to be the origin of the $K=0$ dominance in the PDR strength.

Here, we give another possible mechanism of the $K=0$ dominance in prolate deformed nuclei, which has nothing to do with the neutron skin or the weak binding nature of the valence neutrons. Let us discuss single-particle matrix elements of the dipole operators in the harmonic oscillator potential model. The single-particle states are labeled by the oscillator quanta $\left(n_{x}, n_{y}, n_{z}\right)$ which correspond to the singleparticle energies, $\epsilon_{n_{x}} n_{y} n_{z}=\omega_{x} n_{x}+\omega_{y} n_{y}+\omega_{z} n_{z}+3 / 2$. The well known geometric effect, $\omega_{z}<\omega_{x}$ for a prolate nucleus, lowers the excitation energy of $K=0$ modes $\left(\omega_{z}\right)$ compared to $K=1$ excitations $\left(\omega_{x}=\omega_{y}\right)$. The $K=0$ dominance of the PDR strength can be partially due to this effect, but this is not the only cause. In fact, the deformation in the ground state naturally leads to the $K=0$ enhancement of the dipole matrix elements. It is easy to see that the ratios of available dipole matrix elements between $z$ and $x(y)$ directions are given by

$$
\left|\frac{\left\langle n_{x}, n_{y}, n_{z}+1|z| n_{x}, n_{y}, n_{z}\right\rangle}{\left\langle n_{x}+1, n_{y}, n_{z}|x| n_{x}, n_{y}, n_{z}\right\rangle}\right|^{2}=\frac{n_{z}+1}{n_{x}+1} \frac{\omega_{x}}{\omega_{z}}
$$

For the ratio $|\langle z\rangle /\langle y\rangle|^{2}$, we have the same equation but simply replacing $x$ by $y$. The $K=0$ strength is enhanced by the fact that $\omega_{x} / \omega_{z}=\omega_{y} / \omega_{z}>1$ for prolate nuclei. For strongly deformed $\mathrm{Sr}$ and $\mathrm{Zr}$ isotopes with $\beta_{2} \approx 0.37$, this factor amounts to about 1.5. In addition, for occupied (hole) orbitals, the average value of $n_{z}$ is larger than $n_{x}$. This also contributes to the $K=0$ enhancement.

\section{SYSTEMATIC BEHAVIORS IN PDR STRENGTHS AROUND $N=82$}

It is a well known fact that most of the $E 1$ strengths in nuclei are carried by the GDR at high frequency. Therefore, the low-energy $E 1$ strengths are strongly hindered, typically with a factor of $10^{-5}$ in heavy nuclei. In the terminology of the present time-dependent calculation, the time-dependent residual induced field, $\delta h(t)=\delta h / \delta \rho \cdot \delta \rho(t)$, significantly hinders the low-lying single-particle $E 1$ strength. However, in the present study, we show that the PDR peaks in the neutron-rich nuclei at $N>82$ demonstrate some exceptional cases. Namely, low-energy $E 1$ strengths appearing around $E=5 \mathrm{MeV}$ are completely decoupled from the GDR. Both $E 1$ strengths and peak positions remain the same as those of the corresponding unperturbed (single-particle) peaks. In this section, we demonstrate that these striking behaviors can be universally observed in the PDR peaks in heavy neutron-rich isotopes beyond $N=82$.

\section{A. Choice of smoothing function}

So far, we have used the exponential decay function, $w(t)=$ $e^{-\Gamma t / 2}$, as a smoothing function in Eq. (12). This produces

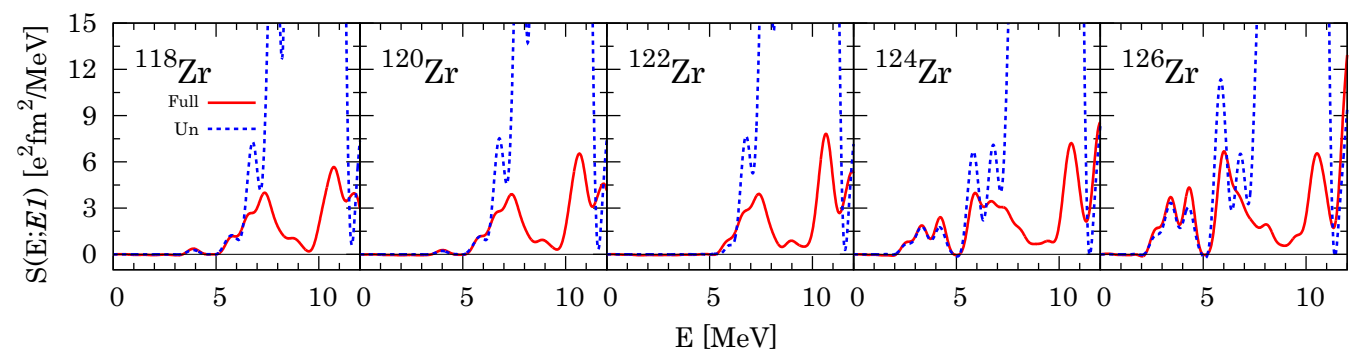

FIG. 20. (Color online) Same as Fig. 19, but for $\mathrm{Zr}$ isotopes. 


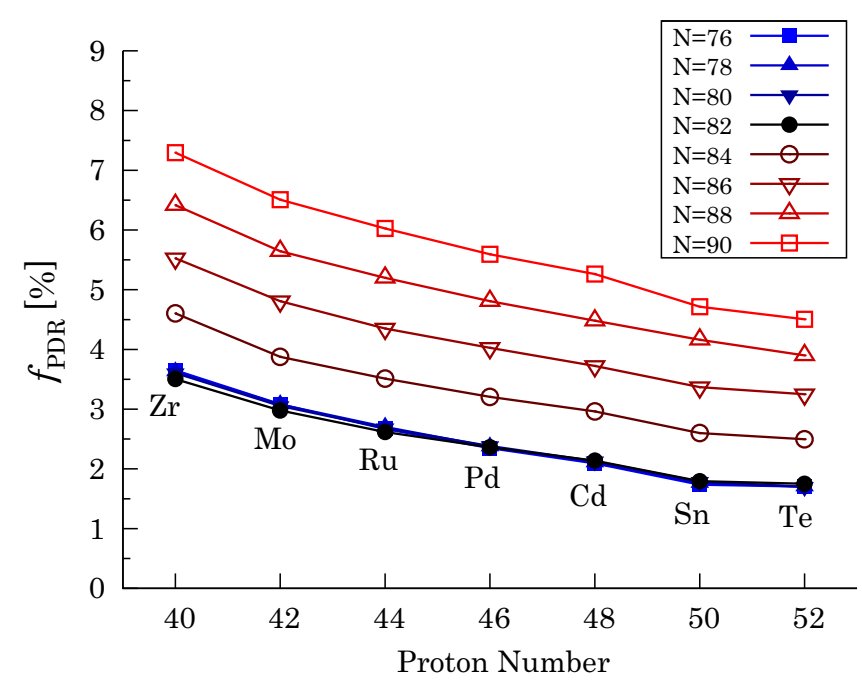

FIG. 21. (Color online) Proton number dependence of the PDR fraction for $\mathrm{Zr}, \mathrm{Mo}, \mathrm{Ru}, \mathrm{Pd}, \mathrm{Cd}, \mathrm{Sn}$, and Te isotopes around $N=82$.

the strength function $S(E ; E 1)$ in which the delta functions in Eq. (11) are smeared by the Lorentzian function of a width $\Gamma$. In order to identify detailed structure of the low-energy $E 1$ peaks, the width of $\Gamma=1 \mathrm{MeV}$ is too large. Each peak has a rather long tail with an asymptotic behavior of $1 /\left|E-E_{n}\right|^{2}$, which makes individual peaks strongly overlap with each other.

Thus, in this section, we use a different smoothing function $w(t)$ in Eq. (12),

$$
w(t)=1-3(t / T)^{2}+2(t / T)^{3} .
$$

This function has properties $w(0)=1, w(T)=0$, and $w^{\prime}(0)=$ $w^{\prime}(T)=0$, which leads to good conservation of the EWSR value in practice. Instead of the Lorentzian function, a peak at $E=E_{n}$ is smeared as

$$
\begin{aligned}
\mathcal{F}\left(E \approx E_{n}\right) & \sim \operatorname{Re} \int_{0}^{T} e^{i\left(E-E_{n}\right) t} w(t) d t \\
& =\frac{6 T}{x^{3}}\left\{-\sin x+\frac{2(1-\cos x)}{x}\right\},
\end{aligned}
$$

where $x=\left(E-E_{n}\right) T$. This smearing function quickly disappears when the energy $E$ is away from the peak position $E_{n}$. In fact, the function becomes zero at $\left|E-E_{n}\right|=2 \pi / T$ and negligibly small at $\left|E-E_{n}\right|>2 \pi / T$.

\section{B. PDR peaks with and without residual fields}

In order to examine the low-energy $E 1$ peaks, we compare the $\mathrm{Cb}$-TDHFB results with the unperturbed results. The unperturbed results can be obtained by neglecting all the residual induced fields. Namely, during the time evolution, we simply ignore the time dependence of mean fields, $h(t)$ and $\Delta(t)$, in Eq. (8), by keeping all the fields same as those in the ground state, $h(t)=h_{0}$ and $\Delta(t)=\Delta_{0}$. The calculated $E 1$ strength functions at $E<12 \mathrm{MeV}$ are shown in Fig. 19 for $\mathrm{Sn}$ isotopes. For $\mathrm{Sn}$ isotopes with $N \leqslant 82\left({ }^{128-132} \mathrm{Sn}\right)$, the $E 1$ strengths appear in the energy region of $E>7 \mathrm{MeV}$. They are almost identical to each other, and are significantly hindered from the unperturbed strengths. This PDR structure at $E=7-12 \mathrm{MeV}$ remains similar even for isotopes beyond $N=82\left({ }^{134-136} \mathrm{Sn}\right)$. However, there appear other low-energy peaks around $E=$ $5 \mathrm{MeV}$. In Fig. 19, the dashed and solid lines coincide with each other around $E=5 \mathrm{MeV}$. These low-energy dipole peaks appearing in $\mathrm{Sn}$ isotopes with $N>82$ are not hindered by the residual effect. In other words, these dipole modes of excitation have pure single-particle nature, being decoupled from the GDR.

A similar feature can be observed more clearly in $\mathrm{Zr}$ isotopes in Fig. 20. The PDR peak around $E=7 \mathrm{MeV}$ exists for all the isotopes in Fig. 20; however, in those with $N>82$, other low-energy peaks emerge around $E=2-5 \mathrm{MeV}$. These new peaks at $N>82$ show no hindrance by the coupling with the GDR.

In Fig. 21, we summarize $f_{\mathrm{PDR}}$ of $\mathrm{Zr}, \mathrm{Mo}, \mathrm{Ru}, \mathrm{Pd}, \mathrm{Cd}$, and Sn isotopes around $N=82$. In each isotopic chain, we find universal behaviors of $f_{\mathrm{PDR}}$ : It stays constant for $76 \leqslant N \leqslant 82$, then jumps up beyond $N=82$. This is due to the fact that two kinds of low-lying dipole modes exist in these isotopes. One of these appears at $E>7 \mathrm{MeV}$ whose $E 1$ strength is strongly hindered by the residual effect. Most probably, it may have

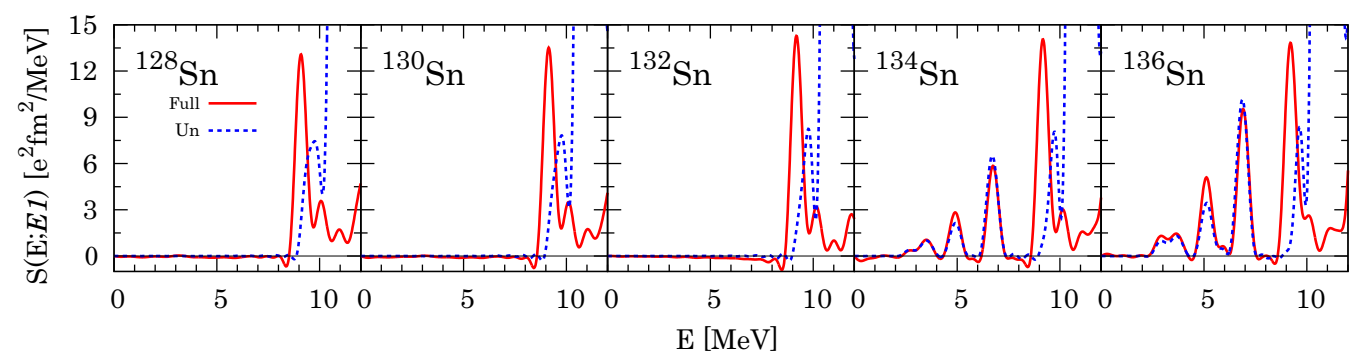

FIG. 22. (Color online) Same as Fig. 19, but with the SkI3 parameter set. 
a collective isoscalar character ${ }^{1}$ [41]. The other one appears at even lower energy of $E=2-7 \mathrm{MeV}$. The $E 1$ strength associated with these peaks shows no hindrance, suggesting that these $E 1$ modes are well decoupled from the GDR.

Finally, in Fig. 22, we show the result of the calculation using the SkI3 functional. There is a prominent PDR peak at $E \approx 9 \mathrm{MeV}$ for all $\mathrm{Sn}$ isotopes for $N=78-86$. In fact, the $E 1$ strength of this peak is enhanced by the residual effect. It means that there is a collective nature of the isovector $(E 1)$ response. This is unusual but explains the property discussed in Sec. IIID that the PDR strength in SkI3 is significantly larger than that in $\mathrm{SkM}^{*}$. Beyond $N=82$, again we can find the PDR peaks at $E=2-7 \mathrm{MeV}$, which show no hindrance by the residual interactions.

\section{CONCLUSION}

We have performed systematic investigation of the $E 1$ modes of excitations in terms of the linear response calculation using the real-time $\mathrm{Cb}$-TDHFB method in the 3D coordinate space representation. In particular, properties of the PDRs and their relations to neutron number, neutron skin thickness, deformation, and chemical potential have been systematically investigated. The present calculation takes into account effects of any kind of deformation and the static and dynamical pairing correlation in the BCS-like approximation. We use the Skyrme energy functional of $\mathrm{SkM}^{*}$ for the present study. For $\mathrm{Sn}$ isotopes, we also adopt the SkI3 parameter set to discuss the origin of known difference between nonrelativistic and relativistic calculations.

We have confirmed a strong neutron shell effect. Especially, we see magic neutron numbers for the sudden enhancement of the PDR strength, such as $N=14 \rightarrow 16, N=28 \rightarrow 30, N=$ $50 \rightarrow 52$, and $N=82 \rightarrow 84$. These characteristic numbers appear in the results both with and without pairing correlation. However, the shell effects are somewhat weakened by the pairing effect for $\mathrm{S}$ and $\mathrm{Ar}$ isotopes around $N=28$, which indicates a gradual increase of the PDR fractions. This is due to the pairing effect of the fractional occupation probability of single-particle orbits.

The pairing correlation also removes some irregular behaviors in the PDR strength in the region of $60<N<74$. Because of this, we can observe a clear indication of the deformation effect. It is most prominent in $\operatorname{Kr}(Z=36)$, $\operatorname{Sr}(Z=38)$, and $\operatorname{Zr}(Z=40)$ isotopes. There are sudden reductions of the PDR strength at the onset and the end of deformation. In strongly deformed nuclei with prolate shape, such as $\mathrm{Sr}$ and $\mathrm{Zr}$ isotopes, the PDR strength is

\footnotetext{
${ }^{1}$ Unfortunately, it is difficult to perform an accurate real-time calculation for the isoscalar dipole modes, because the instantaneous perturbation $V_{\text {ext }}(t)$ inevitably excites the center-of-mass motion. The large amplitude center-of-mass motion leads to a significant error in the Fourier transformation of Eq. (12).
}

dominated by the $K=0$ component, while the neutron skins in these isotopes are smaller in the direction of the symmetry axis than in the perpendicular directions. Thus, the $K=0$ dominance is not associated with the skin thickness. It may be explained, at least partially, by the difference in the single-particle dipole strengths in the deformed mean-field potential.

We have closely examined the characters of the PDR peaks appearing around $N=82$. It turns out that there are two kinds of peaks. The $E 1$ strengths associated with peaks located at $E>7 \mathrm{MeV}$ are very different from the unperturbed strengths. Generally speaking, they are usually hindered from the unperturbed single-particle strengths. On the other hand, the peaks appearing at $E<5 \mathrm{MeV}$ beyond $N=82$ are identical to those in the unperturbed calculation. This suggests the pure single-particle character and the decoupling of the low-energy dipole modes from the GDR. This decoupling phenomenon beyond $N=82$ can be observed for many kinds of isotopes around $\operatorname{Sn}(Z=50)$ and obtained with different energy functionals as well.

It should be noted that properties of the PDRs are sensitive to underlying nuclear structure, such as the single-particle level. For instance, the single-particle level spacings in SkI3 are generally larger than those in $\mathrm{SkM}^{*}$, due to the smaller effective mass. This leads to significant difference in the PDR strengths for $\mathrm{Sn}$ isotopes between $\mathrm{SkM}^{*}$ and SkI3. The relativistic mean-field calculation with the NL3 parameter set [34] produces a result similar to the nonrelativistic calculation with $\mathrm{SkI} 3$, while the point-coupling relativistic model [40] produces one similar to $\mathrm{SkM}^{*}$. Therefore, the different prediction on the PDR properties in the literature may be mostly attributed to the difference in underlying shell structure.

We have observed some correlation between the PDR strength and the neutron-skin thickness; however, its property and quality depend on the mass number, the neutron number, and the adopted energy functional. In contrast, the strong enhancement of the PDR strengths at specific neutron numbers $(N=14 \rightarrow 16, N=28 \rightarrow 30, N=50 \rightarrow 52$, and $N=$ $82 \rightarrow 84)$ and the decoupling phenomenon observed beyond $N=82$ are more robust and universal.

\section{ACKNOWLEDGMENTS}

This work was supported by JSPS KAKENHI Grants No. 24105008, No. 24105006, No. 25287065, and No. 2528706, and by the MEXT SPIRE Field 5. Computational resources were partially provided by the HPCI Systems Research Projects (hp120192) and the RIKEN Integrated Cluster of Clusters (RICC).

\section{APPENDIX: GROUND-STATE PROPERTIES}

In this Appendix, the calculated ground-state properties are presented in Tables $\mathrm{I}-\mathrm{V}$ for even-even isotopes with $6 \leqslant Z \leqslant 20$ and $28 \leqslant Z \leqslant 50$. 
TABLE I. Ground-state properties of even-even isotopes with $Z=6-20$ obtained by the HF+BCS and HF with SkM* calculation: quadrupole deformation parameters $\left(\beta_{2}, \gamma\right)$, pairing gaps for neutrons and protons $\left(\Delta_{n}, \Delta_{p}\right)$, and chemical potentials for neutrons and protons $\left(\lambda_{n}, \lambda_{p}\right)$. In the case of normal phase $(\Delta=0)$, we define the chemical potential as the single-particle energy of the highest occupied orbital, $\lambda_{n}=\epsilon_{N}^{0}$ and $\lambda_{p}=\epsilon_{Z}^{0}$. The pairing gaps and chemical potentials are given in units of MeV.

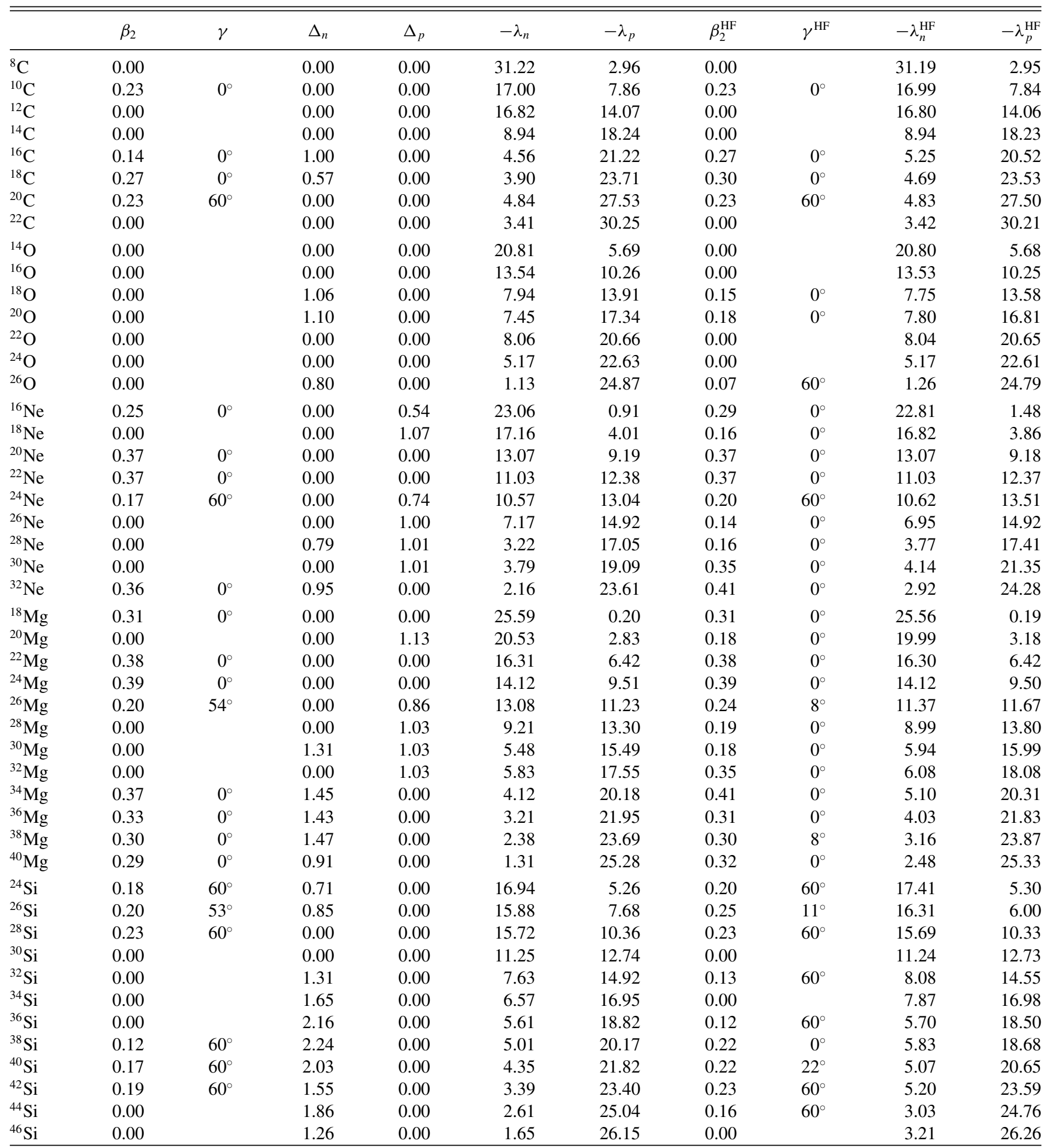


TABLE I. (Continued.)

\begin{tabular}{|c|c|c|c|c|c|c|c|c|c|c|}
\hline & $\beta_{2}$ & $\gamma$ & $\Delta_{n}$ & $\Delta_{p}$ & $-\lambda_{n}$ & $-\lambda_{p}$ & $\beta_{2}^{\mathrm{HF}}$ & $\gamma^{\mathrm{HF}}$ & $-\lambda_{n}^{\mathrm{HF}}$ & $-\lambda_{p}^{\mathrm{HF}}$ \\
\hline${ }^{26} \mathrm{~S}$ & 0.00 & & 1.01 & 0.00 & 18.63 & 1.63 & 0.14 & $0^{\circ}$ & 18.69 & 1.37 \\
\hline${ }^{28} \mathrm{~S}$ & 0.00 & & 1.03 & 0.00 & 17.85 & 3.49 & 0.20 & $0^{\circ}$ & 18.35 & 3.25 \\
\hline${ }^{32} \mathrm{~S}$ & 0.00 & & 0.00 & 0.00 & 13.17 & 7.30 & 0.00 & & 13.15 & 7.29 \\
\hline${ }^{34} \mathrm{~S}$ & 0.00 & & 1.28 & 0.00 & 9.61 & 9.26 & 0.09 & $60^{\circ}$ & 9.86 & 9.27 \\
\hline${ }^{36} \mathrm{~S}$ & 0.00 & & 1.52 & 0.00 & 8.36 & 11.08 & 0.00 & & 9.72 & 11.09 \\
\hline${ }^{40} \mathrm{~S}$ & 0.16 & $0^{\circ}$ & 2.07 & 0.00 & 6.73 & 14.94 & 0.23 & $0^{\circ}$ & 7.77 & 15.24 \\
\hline${ }^{42} \mathrm{~S}$ & 0.15 & $0^{\circ}$ & 2.06 & 0.00 & 5.82 & 16.66 & 0.22 & $0^{\circ}$ & 6.65 & 17.13 \\
\hline${ }^{44} \mathrm{~S}$ & 0.11 & $0^{\circ}$ & 1.97 & 0.00 & 4.88 & 18.00 & 0.10 & $0^{\circ}$ & 5.89 & 18.13 \\
\hline${ }^{46} \mathrm{~S}$ & 0.04 & $1^{\circ}$ & 1.72 & 0.00 & 3.97 & 18.97 & 0.15 & $0^{\circ}$ & 4.57 & 19.74 \\
\hline${ }^{48} \mathrm{~S}$ & 0.00 & & 0.95 & 0.00 & 2.77 & 20.24 & 0.00 & & 4.50 & 20.31 \\
\hline${ }^{50} \mathrm{~S}$ & 0.00 & & 0.00 & 1.72 & 2.21 & 20.98 & 0.00 & & 2.19 & 21.09 \\
\hline${ }^{40} \mathrm{Ar}$ & 0.00 & & 2.03 & 1.22 & 9.27 & 9.93 & 0.12 & $60^{\circ}$ & 9.30 & 10.51 \\
\hline${ }^{42} \mathrm{Ar}$ & 0.00 & & 2.16 & 1.20 & 8.43 & 11.79 & 0.18 & $0^{\circ}$ & 9.17 & 11.73 \\
\hline${ }^{44} \mathrm{Ar}$ & 0.00 & & 1.99 & 1.18 & 7.62 & 13.58 & 0.17 & $17^{\circ}$ & 8.41 & 13.59 \\
\hline${ }^{46} \mathrm{Ar}$ & 0.00 & & 1.77 & 1.16 & 6.49 & 15.20 & 0.14 & $60^{\circ}$ & 8.17 & 16.04 \\
\hline${ }^{48} \mathrm{Ar}$ & 0.00 & & 1.41 & 1.14 & 5.31 & 16.56 & 0.14 & $60^{\circ}$ & 5.73 & 17.29 \\
\hline${ }^{50} \mathrm{Ar}$ & 0.00 & & 0.85 & 2.00 & 3.95 & 17.62 & 0.08 & $60^{\circ}$ & 5.01 & 18.13 \\
\hline${ }^{52} \mathrm{Ar}$ & 0.01 & $59^{\circ}$ & 1.22 & 0.00 & 2.37 & 19.02 & 0.06 & $60^{\circ}$ & 3.08 & 19.03 \\
\hline${ }^{54} \mathrm{Ar}$ & 0.12 & $60^{\circ}$ & 0.00 & 0.00 & 2.22 & 20.96 & 0.12 & $60^{\circ}$ & 2.22 & 20.93 \\
\hline${ }^{56} \mathrm{Ar}$ & 0.00 & & 1.24 & 1.73 & 1.15 & 21.49 & 0.10 & $60^{\circ}$ & 1.42 & 22.27 \\
\hline${ }^{34} \mathrm{Ca}$ & 0.00 & & 0.00 & 0.00 & 21.99 & 1.13 & 0.00 & & 21.97 & 1.12 \\
\hline${ }^{52} \mathrm{Ca}$ & 0.00 & & 1.50 & 0.00 & 5.18 & 17.00 & 0.00 & & 6.67 & 16.95 \\
\hline${ }^{54} \mathrm{Ca}$ & 0.00 & & 2.29 & 0.00 & 4.26 & 18.36 & 0.00 & & 4.11 & 17.99 \\
\hline${ }^{56} \mathrm{Ca}$ & 0.00 & & 2.54 & 1.41 & 3.55 & 17.50 & 0.08 & $60^{\circ}$ & 2.92 & 18.89 \\
\hline${ }^{58} \mathrm{Ca}$ & 0.00 & & 2.54 & 0.00 & 3.01 & 21.12 & 0.08 & $0^{\circ}$ & 3.06 & 20.59 \\
\hline${ }^{60} \mathrm{Ca}$ & 0.00 & & 2.55 & 0.00 & 2.54 & 22.49 & 0.00 & & 3.40 & 22.36 \\
\hline${ }^{62} \mathrm{Ca}$ & 0.00 & & 2.55 & 0.00 & 2.09 & 23.82 & 0.08 & $60^{\circ}$ & 1.92 & 23.04 \\
\hline${ }^{64} \mathrm{Ca}$ & 0.00 & & 2.55 & 0.75 & 1.60 & 22.44 & 0.08 & $0^{\circ}$ & 2.23 & 24.15 \\
\hline
\end{tabular}


TABLE II. Ground-state properties of $\mathrm{Ni}, \mathrm{Zn}, \mathrm{Ge}, \mathrm{Se}$, and $\mathrm{Kr}$ isotopes obtained by the HF+BCS with $\mathrm{SkM}^{*}$ calculation. See the caption of Table I. The root-mean-square radii of neutrons and protons $\left(r_{n}, r_{p}\right)$ are also shown.

\begin{tabular}{|c|c|c|c|c|c|c|c|c|}
\hline & $\beta_{2}$ & $\gamma$ & $r_{n}$ & $r_{p}$ & $\Delta_{n}$ & $\Delta_{p}$ & $-\lambda_{n}$ & $-\lambda_{p}$ \\
\hline $\mathrm{Ni}$ & 0.00 & & 3.63 & 3.69 & 0.00 & 0.36 & 16.27 & 4.31 \\
\hline $\mathrm{Ni}$ & 0.00 & & & & & & & 5.58 \\
\hline $\mathrm{Ni}$ & 0.00 & & 7 & 3.72 & .02 & & & 6.82 \\
\hline $\mathrm{Ni}$ & 0.00 & & 84 & 3.75 & 2.23 & 0.00 & & 10.70 \\
\hline $\mathrm{Ni}$ & 0.00 & & 90 & 3.77 & 2.34 & 0.00 & & 11.91 \\
\hline $5 \mathrm{Ni}$ & 0.00 & & 96 & 3.79 & 2.41 & 0.00 & & 13.11 \\
\hline${ }^{8} \mathrm{Ni}$ & 0.00 & & .01 & 3.82 & 2.27 & 0.00 & & 14.27 \\
\hline $\mathrm{Ni}$ & 0.00 & & 6 & 3.84 & 2.22 & 0.00 & & 15.38 \\
\hline${ }^{2} \mathrm{Ni}$ & 0.00 & & & & & & & 16.50 \\
\hline${ }^{4} \mathrm{Ni}$ & 0.00 & & 4 & 3.8 & 8 & 0. & & 17.58 \\
\hline${ }^{6} \mathrm{Ni}$ & 0.00 & & 0 & 3.89 & 1.70 & 0. & & 18.64 \\
\hline${ }^{8} \mathrm{Ni}$ & 0.00 & & & & & & & 19.69 \\
\hline $\mathrm{Ni}$ & 0.00 & & 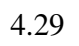 & 3.92 & & 0 & & 20.30 \\
\hline${ }^{2} \mathrm{Ni}$ & 0.00 & & & 3.93 & & & & 20.92 \\
\hline${ }^{84} \mathrm{Ni}$ & 0.00 & & 4.43 & 3.95 & 1.23 & 0.00 & 2.00 & 21.52 \\
\hline${ }^{60} \mathrm{Zn}$ & 0.00 & & 72 & 3.78 & 143 & 096 & 1250 & 3.03 \\
\hline${ }^{62} \mathrm{Zn}$ & 0.01 & $51^{\circ}$ & & & & & & 4.28 \\
\hline${ }^{64} \mathrm{Zn}$ & 0.00 & & 3.85 & 3.82 & 2.15 & .41 & , & 5.51 \\
\hline${ }^{66} \mathrm{Zn}$ & 0.00 & & & & & & & 6.67 \\
\hline $8 \mathrm{Zn}$ & 0.00 & & 2 & 3. & & & & 7.79 \\
\hline${ }^{70} \mathrm{Zn}$ & 0.00 & & 4.0 & & & & & 8.89 \\
\hline${ }^{72} \mathrm{Zn}$ & 0.00 & & 48 & 3. & 2. & & & 9.96 \\
\hline${ }^{74} \mathrm{Zn}$ & 0.01 & $0^{\circ}$ & 4.1 & 3. & 2. & & & 10.98 \\
\hline${ }^{76} \mathrm{Zn}$ & 0.01 & $0^{\circ}$ & 4.1 & 3. & 1. & & & 12.01 \\
\hline${ }^{78} \mathrm{Zn}$ & 0.00 & & 4.1 & & & & & 13.08 \\
\hline${ }^{80} \mathrm{Zn}$ & 0.00 & & 4.2 & & & & & .12 \\
\hline${ }^{82} \mathrm{Zn}$ & 0.00 & & 4.2 & & & & & 14.85 \\
\hline${ }^{84} \mathrm{Zn}$ & 0.01 & $0^{\circ}$ & 4.3 & $3 . C$ & 1.11 & 1. & . & 15.60 \\
\hline${ }^{86} \mathrm{Zn}$ & 0.00 & & 44 & 4.01 & 0 & 1 & 2 & 16.35 \\
\hline${ }^{88} \mathrm{Zn}$ & 0.00 & & 4. & & & & & 17.02 \\
\hline${ }^{64} \mathrm{Ge}$ & 0.0 & & & & & & & 2.34 \\
\hline${ }^{66} \mathrm{Ge}$ & 0.00 & & & & & & & 3.53 \\
\hline${ }^{68} \mathrm{Ge}$ & 0.04 & $59^{\circ}$ & 3. & 3. & 2. & & & 4.95 \\
\hline${ }^{70} \mathrm{Ge}$ & 0.00 & & 3.99 & 3.94 & 2.32 & 7 & 10 & 6.26 \\
\hline${ }^{72} \mathrm{Ge}$ & 0.00 & & 4.04 & & & & & 7.40 \\
\hline${ }^{74} \mathrm{Ge}$ & 0.00 & & & & & & & 3.48 \\
\hline${ }^{76} \mathrm{Ge}$ & 0.12 & $0^{\circ}$ & & & & & & 9.53 \\
\hline${ }^{78} \mathrm{Ge}$ & 0.11 & $0^{\circ}$ & 4.17 & 4.0 & & 1. & & 10.57 \\
\hline${ }^{80} \mathrm{Ge}$ & 0.00 & & 4.20 & 4.01 & 1.54 & 2.16 & & 11.60 \\
\hline${ }^{82} \mathrm{Ge}$ & 0.00 & & 4.23 & 4.02 & 0.00 & 2.17 & 8.74 & 12.62 \\
\hline${ }^{84} \mathrm{Ge}$ & 0.05 & $7^{\circ}$ & 4.29 & 4.04 & 0.96 & 2.06 & 4.78 & 13.38 \\
\hline${ }^{86} \mathrm{Ge}$ & 0.07 & $10^{\circ}$ & 4.36 & 4.06 & 1.18 & 1.95 & 4.26 & 14.15 \\
\hline${ }^{88} \mathrm{Ge}$ & 0.01 & $38^{\circ}$ & 4.41 & & & & & 14.89 \\
\hline${ }^{90} \mathrm{Ge}$ & 0.00 & & 4.48 & & 1.03 & 1.92 & 2.83 & 15.57 \\
\hline${ }^{92} \mathrm{Ge}$ & 0.17 & $28^{\circ}$ & 4.55 & 4.14 & 1.72 & 1.57 & 2.79 & 16.58 \\
\hline${ }^{94} \mathrm{Ge}$ & 0.22 & $26^{\circ}$ & 4.61 & 4.18 & 1.75 & 1.36 & 2.59 & 17.43 \\
\hline${ }^{96} \mathrm{Ge}$ & 0.25 & $22^{\circ}$ & 4.67 & 4.21 & 1.71 & 1.29 & 2.34 & 18.24 \\
\hline${ }^{98} \mathrm{Ge}$ & 0.26 & $18^{\circ}$ & 4.73 & 4.24 & 1.82 & 1.29 & 2.06 & 18.98 \\
\hline
\end{tabular}

TABLE II. (Continued.)

\begin{tabular}{|c|c|c|c|c|c|c|c|c|}
\hline & $\beta_{2}$ & $\gamma$ & $r_{n}$ & $r_{p}$ & $\Delta_{n}$ & $\Delta_{p}$ & $-\lambda_{n}$ & $-\lambda_{p}$ \\
\hline $\mathrm{Se}$ & .09 & $0^{\circ}$ & 3.90 & 3.97 & 2.09 & 1.13 & 12.67 & 2.03 \\
\hline $\mathrm{Se}$ & 18 & $60^{\circ}$ & & & & & & 3.60 \\
\hline $\mathrm{Se}$ & 0.14 & $60^{\circ}$ & 4.02 & 4.02 & 2.13 & & & 4.83 \\
\hline $\mathrm{Se}$ & 0.00 & & 4.05 & 4.02 & 8 & 2.19 & & 5.98 \\
\hline $\mathrm{Se}$ & 0.01 & $46^{\circ}$ & 4.10 & 4.03 & 1.99 & 2.22 & 10.06 & 7.08 \\
\hline $\mathrm{Se}$ & 0.10 & $0^{\circ}$ & 4.15 & 4.05 & 1.93 & 2.15 & & 8.09 \\
\hline $\mathrm{Se}$ & 0.10 & $0^{\circ}$ & 4.18 & 4.06 & 1.68 & 2.16 & & 9.12 \\
\hline $\mathrm{Se}$ & 0.00 & & 4.21 & 4.07 & 1.47 & 2.27 & & 10.20 \\
\hline $\mathrm{Se}$ & 0.00 & & 4.24 & 4.08 & 0.00 & 2.29 & 3 & 11.21 \\
\hline $\mathrm{Se}$ & 0.04 & $0^{\circ}$ & 4.30 & 4.09 & 1.13 & 2.21 & 5 & 11.97 \\
\hline $\mathrm{Se}$ & 0.07 & $0^{\circ}$ & 4.36 & & & & & 12.72 \\
\hline $\mathrm{Se}$ & 0.06 & $60^{\circ}$ & 4.41 & 4. & 1.22 & 2.0 & & 13.49 \\
\hline $\mathrm{Se}$ & 0.10 & $30^{\circ}$ & 4.48 & 4.15 & 1.65 & 1.94 & 3 & 14.26 \\
\hline $\mathrm{Se}$ & 0.20 & $59^{\circ}$ & 4.56 & 4.21 & 1.63 & 1.54 & 6 & 15.16 \\
\hline $\mathrm{Se}$ & 0.33 & $4^{\circ}$ & 4.63 & 4.28 & 1.39 & 1 & ) & 16.26 \\
\hline $\mathrm{Se}$ & 0.32 & $9^{\circ}$ & 4.68 & 4.30 & 1. & & & 16.96 \\
\hline${ }^{100} \mathrm{Se}$ & 0.30 & $0^{\circ}$ & 4.72 & 4. & 4 & & & 17.63 \\
\hline${ }^{102} \mathrm{Se}$ & 0.27 & $0^{\circ}$ & 4.7 & & & & & 3.26 \\
\hline${ }^{04} \mathrm{Se}$ & 0.25 & $0^{\circ}$ & 4.80 & 4.33 & 1.74 & 1.46 & .23 & 18.91 \\
\hline $\mathrm{Kr}$ & 0.26 & $60^{\circ}$ & 403 & 4.11 & 1.78 & 0 & . & 2.15 \\
\hline${ }^{4} \mathrm{Kr}$ & 0.14 & $60^{\circ}$ & 4 & & & & & .55 \\
\hline $\mathrm{Kr}$ & 0.00 & & $4 .($ & 4. & . & 2 & & 4.72 \\
\hline $\mathrm{Kr}$ & 0.00 & & 4.11 & 4.09 & 1. & 2.2 & & 5.80 \\
\hline $\mathrm{Kr}$ & 0.00 & & 4.15 & 4.10 & 1.8 & 23 & & 6.84 \\
\hline $\mathrm{Kr}$ & 0.00 & & 4.19 & 4.11 & 1.' & 2.3 & 10 & 7.86 \\
\hline $\mathrm{Kr}$ & 0.00 & & 4.22 & 4.12 & 1.40 & 2.33 & 6 & 8.86 \\
\hline $\mathrm{Kr}$ & 0.00 & & 4.25 & & 0. & 2.3 & & 9.85 \\
\hline $\mathrm{Kr}$ & 0.01 & $25^{\circ}$ & 4.31 & & & 2.2 & & .62 \\
\hline $\mathrm{Kr}$ & 0.03 & $0^{\circ}$ & 4. & 4. & & & & 11.39 \\
\hline $\mathrm{Kr}$ & 0.00 & & 4.42 & 4.18 & 1. & 2.1 & 2 & 12.18 \\
\hline $\mathrm{Kr}$ & 0.06 & $44^{\circ}$ & 4.47 & 4.20 & 1.63 & 2.04 & 8 & 12.92 \\
\hline $\mathrm{Kr}$ & 0.18 & $60^{\circ}$ & 4.55 & 4.25 & 1. & 1.69 & & 13.64 \\
\hline $\mathrm{Kr}$ & 0.36 & $0^{\circ}$ & 4.64 & 4.35 & 1.37 & 1.41 & 3 & 14.93 \\
\hline${ }^{100} \mathrm{Kr}$ & 0.23 & $60^{\circ}$ & 4.66 & 4.31 & 1.76 & 1.47 & & 15.18 \\
\hline${ }^{102} \mathrm{Kr}$ & 0.35 & $0^{\circ}$ & 4.73 & 4.38 & 1.33 & 1.42 & 3.56 & 16.42 \\
\hline${ }^{104} \mathrm{Kr}$ & 0.32 & $1^{\circ}$ & 4.77 & 4.39 & 1.54 & 1.43 & & 17.02 \\
\hline${ }^{106} \mathrm{Kr}$ & 0.31 & $0^{\circ}$ & 4.80 & 4.40 & 1.51 & 1.43 & 2.87 & 17.63 \\
\hline${ }^{108} \mathrm{Kr}$ & 0.28 & $0^{\circ}$ & 4.84 & 4.41 & 1.48 & 1.42 & 2.55 & 18.18 \\
\hline${ }^{110} \mathrm{Kr}$ & 0.17 & $32^{\circ}$ & 4.84 & 4.38 & 1.86 & 1.57 & 2.50 & 18.88 \\
\hline${ }^{112} \mathrm{Kr}$ & 0.06 & $13^{\circ}$ & 4.84 & 4.38 & 1.78 & 1.78 & 2.54 & 19.90 \\
\hline${ }^{14} \mathrm{Kr}$ & 0.00 & & 4.86 & 4.39 & 1.52 & 1.84 & 2.40 & 20.74 \\
\hline${ }^{116} \mathrm{Kr}$ & 0.00 & & 4.89 & 4.41 & 1.21 & 1.80 & 2.05 & 21.45 \\
\hline${ }^{118} \mathrm{Kr}$ & 0.00 & & 4.92 & 4.43 & 0.00 & 1.77 & 3.72 & 22.14 \\
\hline
\end{tabular}


TABLE III. Same as Table II, but for $\mathrm{Sr}, \mathrm{Zr}$, Mo, and $\mathrm{Ru}$ isotopes.

\begin{tabular}{|c|c|c|c|c|c|c|c|c|}
\hline & $\beta_{2}$ & $\gamma$ & $r_{n}$ & $r_{p}$ & $\Delta_{n}$ & $\Delta_{p}$ & $-\lambda_{n}$ & $-\lambda_{p}$ \\
\hline $\mathrm{Sr}$ & 0.16 & $60^{\circ}$ & 4.06 & 4.14 & 2.05 & 1.59 & 13.41 & 2.17 \\
\hline${ }^{78} \mathrm{Sr}$ & 0.00 & & 4.09 & 4.13 & 1.98 & 2.26 & 12.82 & 3.51 \\
\hline${ }^{80} \mathrm{Sr}$ & 0.00 & & .13 & 4.14 & 1.85 & 2.25 & 12.17 & 4.55 \\
\hline${ }^{82} \mathrm{Sr}$ & 0.00 & & 4.17 & 4.15 & 1.81 & 2.25 & & 5.57 \\
\hline${ }^{84} \mathrm{Sr}$ & 0.00 & & 4.20 & 4.16 & 1.67 & 2.25 & 11.04 & 6.57 \\
\hline${ }^{86} \mathrm{Sr}$ & 0.00 & & 4.23 & 4.17 & 1.35 & 2.24 & 46 & 7.54 \\
\hline${ }^{88} \mathrm{Sr}$ & 0.00 & & 4.26 & 4.18 & 0.00 & 2.24 & 11.65 & 8.50 \\
\hline${ }^{90} \mathrm{Sr}$ & 0.00 & & 4.32 & 4.19 & 1.29 & 2.16 & 7.30 & 9.28 \\
\hline${ }^{92} \mathrm{Sr}$ & 0.00 & & 4.37 & 4.21 & 1.49 & 2.10 & 6.68 & 10.06 \\
\hline${ }^{94} \mathrm{Sr}$ & 0.00 & & 4.42 & 4.22 & 1.66 & 2.05 & 6.10 & 10.84 \\
\hline${ }^{96} \mathrm{Sr}$ & 0.00 & & 4.47 & 4.24 & 1.67 & 2.00 & 5.48 & 11.61 \\
\hline${ }^{98} \mathrm{Sr}$ & 0.37 & $0^{\circ}$ & 4.60 & 4.38 & 1.34 & 1.21 & 5.85 & 12.44 \\
\hline${ }^{100} \mathrm{Sr}$ & 0.38 & $0^{\circ}$ & 4.65 & 4.40 & 1.27 & 1.11 & 5.26 & 13.28 \\
\hline${ }^{102} \mathrm{Sr}$ & 0.38 & $0^{\circ}$ & 4.70 & 4.42 & 1.39 & 1.06 & 4.77 & 14.07 \\
\hline${ }^{104} \mathrm{Sr}$ & 0.37 & $0^{\circ}$ & 4.74 & 4.44 & 1.41 & 1.05 & 4.29 & 14.80 \\
\hline${ }^{106} \mathrm{Sr}$ & 0.36 & $0^{\circ}$ & 4.78 & 4.45 & 1.41 & 1.06 & 3.88 & 15.50 \\
\hline${ }^{108} \mathrm{Sr}$ & 0.35 & $0^{\circ}$ & 4.81 & & 1.25 & & 3.48 & 16.19 \\
\hline${ }^{110} \mathrm{Sr}$ & 0.34 & $0^{\circ}$ & 4.85 & 4.48 & 1.31 & 1.11 & 3.11 & 16.81 \\
\hline${ }^{112} \mathrm{Sr}$ & 0.14 & $60^{\circ}$ & 4.81 & 4.41 & 1.57 & 1.51 & 3.31 & 17.65 \\
\hline${ }^{114} \mathrm{Sr}$ & 0.00 & & 4.83 & 4.41 & 1.61 & 1. & 3.44 & 18.56 \\
\hline${ }^{116} \mathrm{Sr}$ & 0.00 & & 4.86 & 4.43 & 1.56 & & 3. & 19.24 \\
\hline${ }^{118} \mathrm{Sr}$ & 0.00 & & 4.88 & 4.44 & 1.16 & 1.59 & 2.91 & 19.95 \\
\hline${ }^{80} \mathrm{Zr}$ & 0.00 & & 411 & 4.19 & 1.93 & 1.92 & 13.87 & 2.32 \\
\hline${ }^{82} \mathrm{Zr}$ & 0.00 & & & & & & & 3.34 \\
\hline${ }^{84} \mathrm{Zr}$ & 0.00 & & 8 & 4.20 & 1. & & & 4.33 \\
\hline${ }^{86} \mathrm{Zr}$ & 0.00 & & 4.22 & 4.21 & 1.63 & 1.90 & 12. & 5.30 \\
\hline${ }^{88} \mathrm{Zr}$ & 0.00 & & 4.25 & 4.21 & 1.31 & 1.90 & & 6.25 \\
\hline${ }^{90} \mathrm{Zr}$ & 0.00 & & 8 & 4.22 & 0.00 & 1 & 0 & 7.18 \\
\hline${ }^{92} \mathrm{Zr}$ & 0.00 & & & & & & & 7.95 \\
\hline${ }^{94} \mathrm{Zr}$ & 0.00 & & .38 & 4.25 & 1. & 1. & 7. & 8.71 \\
\hline${ }^{96} \mathrm{Zr}$ & 0.00 & & .43 & 4.27 & 1. & & & 9.48 \\
\hline${ }^{98} \mathrm{Zr}$ & 0.00 & & o & 4.28 & 1 & 1. & 5 & 10.24 \\
\hline${ }^{100} \mathrm{Zr}$ & 0.36 & $0^{\circ}$ & 4.60 & 4.41 & 1.35 & 1.35 & 6.61 & 10.76 \\
\hline${ }^{102} \mathrm{Zr}$ & 0.37 & $0^{\circ}$ & 4.65 & 4.44 & 1.32 & 1.26 & 6.06 & 11.48 \\
\hline${ }^{104} \mathrm{Zr}$ & 0.38 & $0^{\circ}$ & 4.70 & 4.46 & 1.33 & & & 12.20 \\
\hline${ }^{106} \mathrm{Zr}$ & 0.38 & $0^{\circ}$ & & & & & & 2.92 \\
\hline${ }^{108} \mathrm{Zr}$ & 0.37 & $0^{\circ}$ & 4.77 & 4.50 & 1.3 & 1. & 4. & 13.66 \\
\hline${ }^{110} \mathrm{Zr}$ & 0.36 & $0^{\circ}$ & 4.8 & & 8 & & & 4.38 \\
\hline${ }^{112} \mathrm{Zr}$ & 0.35 & $0^{\circ}$ & 4.85 & 4.52 & 1.21 & 1. & 7 & 15.08 \\
\hline${ }^{114} \mathrm{Zr}$ & 0.16 & $52^{\circ}$ & 4.81 & 4.46 & 1.56 & 1.36 & 3.98 & 16.24 \\
\hline${ }^{116} \mathrm{Zr}$ & 0.00 & & 4.83 & 4.45 & 1.69 & 1.36 & 4.14 & 16.99 \\
\hline${ }^{118} \mathrm{Zr}$ & 0.00 & & 4.85 & 4.46 & 1.50 & 1.35 & 3.90 & 17.69 \\
\hline${ }^{120} \mathrm{Zr}$ & 0.00 & & & & 1.18 & & & 18.37 \\
\hline${ }^{122} \mathrm{Zr}$ & 0.00 & & 4.90 & 4.49 & 0.00 & 1.35 & 2.93 & 19.07 \\
\hline${ }^{124} \mathrm{Zr}$ & 0.00 & & 4.97 & 4.50 & 1.12 & 1.28 & 1.58 & 19.43 \\
\hline${ }^{126} \mathrm{Zr}$ & 0.00 & & 5.02 & 4.51 & 1.26 & 1.23 & 1.23 & 19.83 \\
\hline${ }^{128} \mathrm{Zr}$ & 0.00 & & 5.08 & 4.52 & 1.31 & 1.17 & 0.91 & 20.23 \\
\hline${ }^{130} \mathrm{Zr}$ & 0.00 & & 5.12 & 4.53 & 0.84 & 1.11 & 0.77 & 20.60 \\
\hline${ }^{84} \mathrm{Mo}$ & 0.00 & & & & & & & 2.17 \\
\hline${ }^{86} \mathrm{Mo}$ & 0.00 & & 4.19 & 4.25 & 1.72 & 1.70 & & 3.14 \\
\hline${ }^{88} \mathrm{Mo}$ & 0.00 & & 4.23 & 4.25 & 1.59 & 1.72 & 12.98 & 4.09 \\
\hline${ }^{90} \mathrm{Mo}$ & 0.00 & & 4.26 & 4.26 & 1.28 & 1.72 & 12.40 & 5.02 \\
\hline${ }^{92} \mathrm{Mo}$ & 0.00 & & 4.29 & 4.26 & 0.00 & 1.74 & 13.48 & 5.95 \\
\hline${ }^{94} \mathrm{Mo}$ & 0.00 & & 4.34 & 4.28 & 1.32 & 1.69 & 8.88 & 6.69 \\
\hline${ }^{96} \mathrm{Mo}$ & 0.00 & & 4.39 & 4.29 & 1.58 & 1.64 & 8.24 & 7.44 \\
\hline${ }^{98} \mathrm{Mo}$ & 0.00 & & 4.43 & 4.31 & 1.71 & 1.59 & 7.64 & 8.20 \\
\hline${ }^{100} \mathrm{Mo}$ & 0.00 & & 4.48 & 4.33 & 1.67 & 1.55 & 7.02 & 8.95 \\
\hline
\end{tabular}

TABLE III. (Continued.)

\begin{tabular}{|c|c|c|c|c|c|c|c|c|}
\hline & $\beta_{2}$ & $\gamma$ & $r_{n}$ & $r_{p}$ & $\Delta_{n}$ & $\Delta_{p}$ & $-\lambda_{n}$ & $-\lambda_{p}$ \\
\hline${ }^{102} \mathrm{Mo}$ & 0.29 & $12^{\circ}$ & 4.58 & 4.42 & 1.55 & 1.43 & 7.20 & 9.67 \\
\hline${ }^{104} \mathrm{Mo}$ & 0.32 & $12^{\circ}$ & 4.63 & 4.45 & 1.42 & 1.33 & 81 & 10.33 \\
\hline${ }^{106} \mathrm{Mo}$ & 0.33 & $14^{\circ}$ & 4.68 & 4.48 & 1.36 & 1.27 & 6.33 & 11.07 \\
\hline${ }^{108} \mathrm{Mo}$ & 0.32 & $17^{\circ}$ & 4.72 & 4.49 & 1.35 & 1.22 & 5.87 & 11.87 \\
\hline${ }^{110} \mathrm{Mo}$ & 0.30 & $19^{\circ}$ & 4.75 & 4.50 & 1.38 & 1.22 & 5.44 & 12.66 \\
\hline${ }^{112} \mathrm{Mo}$ & 0.28 & $23^{\circ}$ & 4.78 & 4.51 & 1.38 & 1.26 & 5.07 & 13.47 \\
\hline${ }^{114} \mathrm{Mo}$ & 0.24 & $27^{\circ}$ & 4.80 & 4.51 & 1.39 & 1.30 & 4.79 & 14.26 \\
\hline${ }^{116} \mathrm{Mo}$ & 0.20 & $33^{\circ}$ & 4.82 & 4.51 & 1.32 & 1.29 & 4.56 & 14.96 \\
\hline${ }^{118} \mathrm{Mo}$ & 0.00 & & 4.82 & 4.48 & 1.64 & 1.36 & 4.86 & 15.67 \\
\hline${ }^{120} \mathrm{Mo}$ & 0.00 & & 4.85 & 4.50 & 1.46 & 1.35 & 4.61 & 16.37 \\
\hline${ }^{122} \mathrm{Mo}$ & 0.00 & & 4.87 & 4.51 & 1.14 & 1.36 & 4.32 & 17.06 \\
\hline${ }^{124} \mathrm{Mo}$ & 0.00 & & 4.90 & 4.53 & 0.00 & 1.36 & 5.75 & 17.76 \\
\hline${ }^{126} \mathrm{Mo}$ & 0.00 & & 4.95 & 4.54 & 0.70 & 1.31 & 1.78 & 18.19 \\
\hline${ }^{128} \mathrm{Mo}$ & 0.00 & & 5.00 & 4.55 & 0.98 & 1.27 & 1.63 & 18.56 \\
\hline${ }^{130} \mathrm{Mo}$ & 0.00 & & 5.05 & 4.56 & 0.97 & 1.22 & 1.37 & 18.95 \\
\hline${ }^{132} \mathrm{Mo}$ & 0.00 & & 5.10 & 4.57 & 0.89 & 1.19 & 1.08 & 19.32 \\
\hline${ }^{8} \mathrm{Ru}$ & 0.00 & & 4.21 & 4.29 & 1.69 & 1.64 & 14.50 & 2.05 \\
\hline${ }^{90} \mathrm{Ru}$ & 0.00 & & 4.24 & 4.29 & 1.56 & 1.65 & 13.93 & 2.99 \\
\hline${ }^{92} \mathrm{Ru}$ & 0.00 & & 4.27 & 4.30 & 1.25 & 1.66 & 13.33 & 3.90 \\
\hline${ }^{94} \mathrm{Ru}$ & 0.00 & & 4.30 & 4.30 & 0.00 & 1.67 & 14.38 & 4.81 \\
\hline${ }^{96} \mathrm{Ru}$ & 0.00 & & 4.35 & 4.32 & 1.33 & 1.61 & 9.64 & 5.56 \\
\hline${ }^{98} \mathrm{Ru}$ & 0.00 & & 4.40 & 4.34 & 1.62 & 1.60 & 9.00 & 6.30 \\
\hline${ }^{100} \mathrm{Ru}$ & 0.00 & & 4.44 & 4.35 & 1.74 & 1.56 & 10 & 7.06 \\
\hline${ }^{102} \mathrm{Ru}$ & 0.00 & & 4.48 & 4.37 & 1.72 & 1.53 & 7.80 & 7.81 \\
\hline${ }^{104} \mathrm{Ru}$ & 0.13 & $0^{\circ}$ & 4.54 & 4.40 & 1.85 & 1.52 & 7.58 & 8.73 \\
\hline${ }^{106} \mathrm{Ru}$ & 0.27 & $18^{\circ}$ & 4.62 & 4.47 & 1.60 & 1.19 & 7.49 & 9.07 \\
\hline${ }^{108} \mathrm{Ru}$ & 0.28 & $19^{\circ}$ & 4.66 & 4.49 & 1.53 & 1.13 & 7.08 & 9.76 \\
\hline${ }^{110} \mathrm{Ru}$ & 0.28 & $21^{\circ}$ & 4.70 & 4.51 & 1.49 & 1.08 & 6.66 & 10.51 \\
\hline${ }^{112} \mathrm{Ru}$ & 0.27 & $23^{\circ}$ & 4.74 & 4.52 & 1.45 & 1.04 & 6.24 & 11.28 \\
\hline${ }^{114} \mathrm{Ru}$ & 0.25 & $26^{\circ}$ & 4.77 & 4.53 & 1.41 & 1.02 & 5.85 & 12.05 \\
\hline${ }^{116} \mathrm{Ru}$ & 0.23 & $27^{\circ}$ & 4.79 & 4.54 & 1.38 & 1.04 & 5.51 & 12.81 \\
\hline${ }^{118} \mathrm{Ru}$ & 0.04 & $1^{\circ}$ & 4.79 & 4.51 & 1.76 & 1.37 & 5.75 & 13.85 \\
\hline${ }^{120} \mathrm{Ru}$ & 0.00 & & 4.82 & 4.52 & 1.63 & 1.36 & 5.56 & 14.54 \\
\hline${ }^{122} \mathrm{Ru}$ & 0.00 & & 4.84 & 4.53 & 1.43 & 1.36 & 5.31 & 15.24 \\
\hline${ }^{124} \mathrm{Ru}$ & 0.00 & & 4.87 & 4.55 & 1.11 & 1.35 & 5.02 & 15.93 \\
\hline${ }^{126} \mathrm{Ru}$ & 0.00 & & 4.89 & 4.56 & 0.00 & 1.36 & 6.44 & 16.62 \\
\hline${ }^{128} \mathrm{Ru}$ & 0.00 & & 4.94 & 4.57 & 0.75 & 1.31 & 2.29 & 17.04 \\
\hline${ }^{130} \mathrm{Ru}$ & 0.00 & & 4.99 & 4.59 & 0.83 & 1.29 & 2.04 & 17.46 \\
\hline${ }^{132} \mathrm{Ru}$ & 0.00 & & 5.04 & 4.60 & 0.89 & 1.25 & 1.80 & 17.85 \\
\hline${ }^{134} \mathrm{Ru}$ & 0.00 & & 5.09 & 4.61 & 0.80 & 1.23 & 1.48 & 18.24 \\
\hline
\end{tabular}


TABLE IV. Same as Table II, but for Pd and Cd isotopes.

\begin{tabular}{|c|c|c|c|c|c|c|c|c|}
\hline & $\beta_{2}$ & $\gamma$ & $r_{n}$ & $r_{p}$ & $\Delta_{n}$ & $\Delta_{p}$ & $-\lambda_{n}$ & $-\lambda_{p}$ \\
\hline${ }^{92} \mathrm{Pd}$ & 0.00 & & 4.25 & 4.33 & 1.54 & 1.49 & 14.85 & 1.94 \\
\hline${ }^{4} \mathrm{Pd}$ & 0.00 & & 4.28 & 4.34 & 1.23 & 1.49 & 14.25 & 2.84 \\
\hline${ }^{96} \mathrm{Pd}$ & 0.00 & & 4.31 & 4.34 & 0.00 & 1.50 & 15.27 & 3.73 \\
\hline${ }^{98} \mathrm{Pd}$ & 0.00 & & 4.36 & 4.36 & 1.34 & 1.46 & 10.39 & 4.48 \\
\hline${ }^{100} \mathrm{Pd}$ & 0.00 & & 4.40 & 4.37 & 1.65 & 1.42 & 9.75 & 5.24 \\
\hline${ }^{102} \mathrm{Pd}$ & 0.00 & & 4.45 & 4.39 & 1.76 & 1.43 & 9.15 & 5.99 \\
\hline${ }^{104} \mathrm{Pd}$ & 0.08 & $0^{\circ}$ & 4.50 & 4.41 & 1.82 & 1.33 & 8.68 & 6.75 \\
\hline${ }^{106} \mathrm{Pd}$ & 0.14 & $0^{\circ}$ & 4.55 & 4.44 & 1.85 & 1.19 & 8.37 & 7.51 \\
\hline${ }^{108} \mathrm{Pd}$ & 0.17 & $0^{\circ}$ & 4.60 & 4.47 & 1.87 & 1.13 & 8.05 & 8.23 \\
\hline${ }^{110} \mathrm{Pd}$ & 0.19 & $0^{\circ}$ & 4.64 & 4.49 & 1.85 & 1.10 & 7.72 & 8.93 \\
\hline${ }^{112} \mathrm{Pd}$ & 0.20 & $0^{\circ}$ & 4.68 & 4.51 & 1.79 & 1.08 & 7.38 & 9.64 \\
\hline${ }^{114} \mathrm{Pd}$ & 0.19 & $0^{\circ}$ & 4.71 & 4.52 & 1.71 & 1.07 & 7.04 & 10.39 \\
\hline${ }^{116} \mathrm{Pd}$ & 0.18 & $0^{\circ}$ & 4.74 & 4.53 & 1.65 & 106 & 6.71 & 1.17 \\
\hline${ }^{118} \mathrm{Pd}$ & 0.15 & $0^{\circ}$ & 4.77 & 4.54 & 1.62 & 1.06 & 6.46 & 11.95 \\
\hline${ }^{120} \mathrm{Pd}$ & 0.10 & $0^{\circ}$ & 4.79 & 4.55 & 1.66 & 1.11 & 6.34 & 12.73 \\
\hline${ }^{122} \mathrm{Pd}$ & 0.00 & & 4.81 & 4.55 & 1.62 & 1.26 & 6.26 & 13.47 \\
\hline${ }^{124} \mathrm{Pd}$ & 0.00 & & 4.84 & 4.57 & 1.43 & 1.25 & 6.00 & 14.17 \\
\hline${ }^{126} \mathrm{Pd}$ & 0.00 & & 4.87 & 4.58 & 1.10 & 1.24 & 5.69 & 14.85 \\
\hline${ }^{128} \mathrm{Pd}$ & 0.00 & & 4.89 & 4.59 & 0.00 & 1.24 & 7.12 & 15.53 \\
\hline${ }^{130} \mathrm{Pd}$ & 0.00 & & 4.94 & 4.61 & 0.71 & 1.21 & 2.72 & 15.96 \\
\hline${ }^{132} \mathrm{Pd}$ & 0.00 & & 4.98 & 4.62 & 0.80 & 1.19 & 2.47 & 16.40 \\
\hline${ }^{134} \mathrm{Pd}$ & 0.00 & & 5.03 & 4.63 & 0.75 & 1.16 & 2.23 & 16.84 \\
\hline${ }^{136} \mathrm{Pd}$ & 0.00 & & 5.07 & 4.64 & 0.60 & 1.15 & 1.86 & 17.25 \\
\hline${ }^{96} \mathrm{Cd}$ & 0.00 & & 4.29 & 4.38 & 1 & 1.1 & 15.16 & 1.79 \\
\hline${ }^{98} \mathrm{Cd}$ & 0.00 & & 4.32 & 4.38 & 0.00 & 1.18 & 16. & 2.66 \\
\hline${ }^{100} \mathrm{Cd}$ & 0.00 & & 4.37 & 4.40 & 1.35 & 1.14 & 11. & 3.42 \\
\hline${ }^{102} \mathrm{Cd}$ & 0.00 & & 4.41 & 4.41 & 1. & 1.11 & 10. & 4.19 \\
\hline${ }^{104} \mathrm{Cd}$ & 0.00 & & 4.45 & 4.43 & 1.78 & 1.13 & 9.90 & 4.92 \\
\hline${ }^{106} \mathrm{Cd}$ & 0.00 & & 4.50 & 4.44 & 1.80 & & 6 & 5.69 \\
\hline${ }^{108} \mathrm{Cd}$ & 0.09 & $0^{\circ}$ & 4.55 & 4.47 & 1.91 & 0. & 9.07 & 6.31 \\
\hline${ }^{110} \mathrm{Cd}$ & 0.13 & $0^{\circ}$ & 4.59 & 4.49 & 1.91 & 0.76 & 5 & 6.89 \\
\hline${ }^{112} \mathrm{Cd}$ & 0.15 & $0^{\circ}$ & 4.63 & 4.51 & 1.91 & 0.64 & 3 & 7.52 \\
\hline${ }^{114} \mathrm{Cd}$ & 0.16 & $0^{\circ}$ & 4.67 & 4.53 & 1.87 & 0.55 & 8.11 & 8.17 \\
\hline${ }^{116} \mathrm{Cd}$ & 0.16 & $0^{\circ}$ & 4.70 & 4.55 & 1.82 & 0.49 & 7.79 & 8.88 \\
\hline${ }^{118} \mathrm{Cd}$ & 0.15 & $0^{\circ}$ & 4.74 & 4.56 & 1.75 & 0.50 & 7.49 & 9.66 \\
\hline${ }^{120} \mathrm{Cd}$ & 0.14 & $0^{\circ}$ & 4.77 & 4.57 & 1.64 & 0.00 & 7.19 & 11.33 \\
\hline${ }^{122} \mathrm{Cd}$ & 0.00 & & 4.78 & 4.57 & 1.75 & 1.00 & 7.19 & 11.72 \\
\hline${ }^{124} \mathrm{Cd}$ & 0.00 & & 4.81 & 4.59 & 1.62 & 0.99 & 6.94 & 12.41 \\
\hline${ }^{126} \mathrm{Cd}$ & 0.00 & & 4.84 & 4.60 & 1.42 & 0.98 & 6.67 & 13.10 \\
\hline${ }^{128} \mathrm{Cd}$ & 0.00 & & 4.87 & 4.61 & 1.10 & 0.98 & 6.36 & 13.78 \\
\hline${ }^{130} \mathrm{Cd}$ & 0.00 & & 4.89 & 4.63 & 0.00 & 0.97 & 7.80 & 14.45 \\
\hline${ }^{132} \mathrm{Cd}$ & 0.00 & & 4.93 & 4.64 & 0.64 & 0.95 & 3.13 & 14.91 \\
\hline${ }^{134} \mathrm{Cd}$ & 0.00 & & 4.98 & 4.65 & 0.72 & 0.94 & 2.90 & 15.36 \\
\hline${ }^{136} \mathrm{Cd}$ & 0.00 & & 5.02 & 4.66 & 0.76 & 0.92 & 2.66 & 15.80 \\
\hline${ }^{138} \mathrm{Cd}$ & 0.00 & & 5.06 & 4.68 & 0.59 & 0.91 & 2.24 & 16.23 \\
\hline
\end{tabular}

TABLE V. Ground-state properties of Sn isotopes calculated with $\mathrm{SkM}^{*}$ and SkI3. See the captions of Tables I and II.

\begin{tabular}{|c|c|c|c|c|c|c|c|c|}
\hline $\mathrm{SkM}^{*}$ & $\beta_{2}$ & $\gamma$ & $r_{n}$ & $r_{p}$ & $\Delta_{n}$ & $\Delta_{p}$ & $-\lambda_{n}$ & $-\lambda_{p}$ \\
\hline${ }^{100} \mathrm{Sn}$ & 0.00 & & 4.33 & 4.42 & 0.00 & 0.00 & 17.00 & 3.15 \\
\hline${ }^{102} \mathrm{Sn}$ & 0.00 & & 4.38 & 4.43 & 1.35 & 0.00 & 11.86 & 3.86 \\
\hline${ }^{104} \mathrm{Sn}$ & 0.00 & & 4.42 & 4.45 & 1.70 & 0.00 & 11.24 & 4.59 \\
\hline${ }^{106} \mathrm{Sn}$ & 0.00 & & 4.46 & 4.46 & 1.80 & 0.00 & 10.65 & 5.33 \\
\hline${ }^{108} \mathrm{Sn}$ & 0.00 & & 4.50 & 4.48 & 1.84 & 0.00 & 10.13 & 6.07 \\
\hline${ }^{110} \mathrm{Sn}$ & 0.00 & & 4.54 & 4.49 & 1.97 & 0.00 & 9.75 & 6.84 \\
\hline${ }^{112} \mathrm{Sn}$ & 0.00 & & 4.58 & 4.51 & 2.05 & 0.00 & 9.43 & 7.60 \\
\hline${ }^{114} \mathrm{Sn}$ & 0.00 & & 4.62 & 4.53 & 2.10 & 0.00 & 9.13 & 8.35 \\
\hline${ }^{116} \mathrm{Sn}$ & 0.00 & & 4.66 & 4.55 & 2.06 & 0.00 & 8.86 & 9.09 \\
\hline${ }^{118} \mathrm{Sn}$ & 0.00 & & 4.69 & 4.56 & 2.00 & 0.00 & 8.60 & 9.82 \\
\hline${ }^{120} \mathrm{Sn}$ & 0.00 & & 4.72 & 4.58 & 1.94 & 0.00 & 8.36 & 10.54 \\
\hline${ }^{122} \mathrm{Sn}$ & 0.00 & & 4.75 & 4.59 & 1.85 & 0.00 & 8.12 & 11.25 \\
\hline${ }^{124} \mathrm{Sn}$ & 0.00 & & 4.78 & 4.61 & 1.75 & 0.00 & 7.87 & 11.95 \\
\hline${ }^{126} \mathrm{Sn}$ & 0.00 & & 4.81 & 4.62 & 1.62 & 0.00 & 7.62 & 12.63 \\
\hline${ }^{128} \mathrm{Sn}$ & 0.00 & & 4.84 & 4.63 & 1.42 & 0.00 & 7.34 & 13.31 \\
\hline${ }^{130} \mathrm{Sn}$ & 0.00 & & 4.87 & 4.64 & 1.10 & 0.00 & 7.01 & 13.98 \\
\hline${ }^{132} \mathrm{Sn}$ & 0.00 & & 4.89 & 4.66 & 0.00 & 0.00 & 8.48 & 14.64 \\
\hline${ }^{134} \mathrm{Sn}$ & 0.00 & & 4.93 & 4.67 & 0.68 & 0.00 & 3.62 & 15.07 \\
\hline${ }^{136} \mathrm{Sn}$ & 0.00 & & 4.97 & 4.68 & 0.78 & 0.00 & 3.36 & 15.51 \\
\hline${ }^{138} \mathrm{Sn}$ & 0.00 & & 5.02 & 4.69 & 1.04 & 0.00 & 3.11 & 15.90 \\
\hline${ }^{140} \mathrm{Sn}$ & 0.00 & & 5.07 & 4.71 & 1.74 & 0.00 & 3.06 & 16.43 \\
\hline SkI3 & $\beta_{2}$ & $\gamma$ & $r_{n}$ & $r_{p}$ & $\Delta_{n}$ & $\Delta_{p}$ & $-\lambda_{n}$ & $-\lambda_{p}$ \\
\hline$\overline{{ }^{100} \mathrm{Sn}}$ & 0.00 & & 4.33 & 4.40 & 0.00 & 0.00 & 17.18 & 3.50 \\
\hline${ }^{102} \mathrm{Sn}$ & 0.00 & & 4.38 & 4.42 & 1.54 & 0.00 & 11.00 & 4.33 \\
\hline${ }^{104} \mathrm{Sn}$ & 0.00 & & 4.43 & 4.43 & 1.65 & 0.00 & 10.40 & 5.16 \\
\hline${ }^{106} \mathrm{Sn}$ & 0.00 & & 4.48 & 4.45 & 2.20 & 0.00 & 10.34 & 6.00 \\
\hline${ }^{108} \mathrm{Sn}$ & 0.00 & & 4.52 & 4.47 & 1.83 & 0.00 & 9.93 & 6.81 \\
\hline${ }^{110} \mathrm{Sn}$ & 0.00 & & 4.56 & 4.49 & 2.37 & 0.00 & 9.76 & 7.61 \\
\hline${ }^{112} \mathrm{Sn}$ & 0.00 & & 4.60 & 4.50 & 2.43 & 0.00 & 9.41 & 8.38 \\
\hline${ }^{114} \mathrm{Sn}$ & 0.00 & & 4.64 & 4.52 & 2.31 & 0.00 & 9.09 & 9.13 \\
\hline${ }^{116} \mathrm{Sn}$ & 0.00 & & 4.67 & 4.53 & 2.02 & 0.00 & 8.69 & 9.85 \\
\hline${ }^{118} \mathrm{Sn}$ & 0.00 & & 4.71 & 4.55 & 2.04 & 0.00 & 8.28 & 10.55 \\
\hline${ }^{120} \mathrm{Sn}$ & 0.00 & & 4.75 & 4.56 & 0.00 & 0.00 & 8.50 & 10.94 \\
\hline${ }^{122} \mathrm{Sn}$ & 0.00 & & 4.78 & 4.57 & 1.70 & 0.00 & 7.61 & 11.94 \\
\hline${ }^{124} \mathrm{Sn}$ & 0.00 & & 4.81 & 4.59 & 1.68 & 0.00 & 7.34 & 12.67 \\
\hline${ }^{126} \mathrm{Sn}$ & 0.00 & & 4.84 & 4.60 & 1.61 & 0.00 & 7.10 & 13.40 \\
\hline${ }^{128} \mathrm{Sn}$ & 0.00 & & 4.87 & 4.61 & 1.46 & 0.00 & 6.85 & 14.13 \\
\hline${ }^{130} \mathrm{Sn}$ & 0.00 & & 4.90 & 4.62 & 1.19 & 0.00 & 6.54 & 14.86 \\
\hline${ }^{132} \mathrm{Sn}$ & 0.00 & & 4.93 & 4.64 & 0.00 & 0.00 & 8.00 & 15.56 \\
\hline${ }^{134} \mathrm{Sn}$ & 0.00 & & 4.99 & 4.65 & 0.78 & 0.00 & 2.00 & 15.93 \\
\hline${ }^{136} \mathrm{Sn}$ & 0.00 & & 5.04 & 4.67 & 1.30 & 0.00 & 2.10 & 16.39 \\
\hline${ }^{138} \mathrm{Sn}$ & 0.00 & & 5.10 & 4.69 & 1.61 & 0.00 & 2.09 & 16.63 \\
\hline${ }^{140} \mathrm{Sn}$ & 0.00 & & 5.14 & 4.71 & 1.66 & 0.00 & 1.94 & 17.22 \\
\hline
\end{tabular}


[1] A. Leistenschneider et al., Phys. Rev. Lett. 86, 5442 (2001).

[2] E. Tryggestad et al., Phys. Rev. C 67, 064309 (2003).

[3] J. Gibelin et al., Phys. Rev. Lett. 101, 212503 (2008).

[4] T. Hartmann, M. Babilon, S. Kamerdzhiev, E. Litvinova, D. Savran, S. Volz, and A. Zilges, Phys. Rev. Lett. 93, 192501 (2004).

[5] J. Isaak et al., Phys. Rev. C 83, 034304 (2011).

[6] F. Bauwens et al., Phys. Rev. C 62, 024302 (2000).

[7] O. Wieland et al., Phys. Rev. Lett. 102, 092502 (2009).

[8] L. Käubler et al., Phys. Rev. C 70, 064307 (2004).

[9] R. Schwengner et al., Phys. Rev. C 78, 064314 (2008).

[10] C. Iwamoto et al., Phys. Rev. Lett. 108, 262501 (2012).

[11] K. Govaert et al., Phys. Rev. C 57, 2229 (1998).

[12] P. Adrich et al., Phys. Rev. Lett. 95, 132501 (2005).

[13] B. Özel et al., Nucl. Phys. A 788, 385 (2007).

[14] A. Klimkiewicz et al., Phys. Rev. C 76, 051603(R) (2007).

[15] J. Endres et al., Phys. Rev. Lett. 105, 212503 (2010).

[16] R.-D. Herzberg et al., Phys. Rev. C 60, 051307 (1999).

[17] A. Zilges et al., Nucl. Phys. A 731, 249 (2004).

[18] S. Volz et al., Nucl. Phys. A 779, 1 (2006).

[19] D. Savran et al., Phys. Rev. C 84, 024326 (2011).

[20] J. Endres et al., Phys. Rev. C 80, 034302 (2009).

[21] A. P. Tonchev et al., Phys. Rev. Lett. 104, 072501 (2010).

[22] T. Chapuran, R. Vodhanel, and M. K. Brussel, Phys. Rev. C 22, 1420 (1980).

[23] N. Ryezayeva et al., Phys. Rev. Lett. 89, 272502 (2002).

[24] J. Enders et al., Nucl. Phys. A 724, 243 (2003).

[25] A. Tamii et al., Phys. Rev. Lett. 107, 062502 (2011).

[26] I. Poltoratska et al., Phys. Rev. C 85, 041304(R) (2012).

[27] G. Colò and P. F. Bortignon, Nucl. Phys. A 696, 427 (2001).

[28] J. Terasaki, J. Engel, M. Bender, J. Dobaczewski, W. Nazarewicz, and M. Stoitsov, Phys. Rev. C 71, 034310 (2005).

[29] S. Ebata, T. Nakatsukasa, T. Inakura, K. Yoshida, Y. Hashimoto, and K. Yabana, Phys. Rev. C 82, 034306 (2010).

[30] E. Yüksel, E. Khan, and K. Bozkurt, Nucl. Phys. A 877, 35 (2012).

[31] D. Vretenar, N. Paar, P. Ring, and G. A. Lalazissis, Nucl. Phys. A 692, 496 (2001).

[32] N. Paar, P. Ring, T. Nikšić, and D. Vretenar, Phys. Rev. C 67, 034312 (2003).

[33] L.-G. Cao and Z.-Y. Ma, Phys. Rev. C 71, 034305 (2005).

[34] J. Piekarewicz, Phys. Rev. C 73, 044325 (2006).

[35] J. Liang, L.-G. Cao, and Z.-Y. Ma, Phys. Rev. C 75, 054320 (2007).

[36] D. Peña Artega and P. Ring, Phys. Rev. C 77, 034317 (2008).

[37] H. Nakada, K. Mizuyama, M. Yamagami, and M. Matsuo, Nucl. Phys. A 828, 283 (2009).

[38] C. Losa, A. Pastore, T. Døssing, E. Vigezzi, and R. A. Broglia, Phys. Rev. C 81, 064307 (2010).

[39] J. Piekarewicz, Phys. Rev. C 83, 034319 (2011).

[40] I. Daoutidis and P. Ring, Phys. Rev. C 83, 044303 (2011).

[41] X. Roca-Maza, G. Pozzi, M. Brenna, K. Mizuyama, and G. Colò, Phys. Rev. C 85, 024601 (2012).

[42] A. Carbone, G. Colò, A. Bracco, L.-G. Cao, P. F. Bortignon, F. Camera, and O. Wieland, Phys. Rev. C 81, 041301(R) (2010).

[43] S. Péru, J. Berger, and P. Bortignon, Eur. Phys. J. A 26, 25 (2005).

[44] D. Sarchi, P. Bortignon, and G. Colò, Phys. Lett. B 601, 27 (2004).
[45] N. Tsoneva, H. Lenske, and C. Stoyanov, Nucl. Phys. A 731, 273 (2004).

[46] E. Litvinova, P. Ring, V. Tselyaev, and K. Langanke, Phys. Rev. C 79, 054312 (2009).

[47] A. Avdeenkov, S. Goriely, S. Kamerdzhiev, and S. Krewald, Phys. Rev. C 83, 064316 (2011).

[48] D. Gambacurta, M. Grasso, and F. Catara, Phys. Rev. C 84, 034301 (2011).

[49] M. Tohyama and T. Nakatsukasa, Phys. Rev. C 85, 031302 (2012).

[50] T. Nakatsukasa, T. Inakura, and K. Yabana, Phys. Rev. C 76, 024318 (2007).

[51] T. Inakura, T. Nakatsukasa, and K. Yabana, Phys. Rev. C 80, 044301 (2009).

[52] P. Avogadro and T. Nakatsukasa, Phys. Rev. C 84, 014314 (2011).

[53] M. Stoitsov, M. Kortelainen, T. Nakatsukasa, C. Losa, and W. Nazarewicz, Phys. Rev. C 84, 041305 (2011).

[54] P. Avogadro and T. Nakatsukasa, Phys. Rev. C 87, 014331 (2013).

[55] H. Liang, T. Nakatsukasa, Z. Niu, and J. Meng, Phys. Rev. C 87, 054310 (2013).

[56] N. Hinohara, M. Kortelainen, and W. Nazarewicz, Phys. Rev. C 87, 064309 (2013).

[57] T. Inakura, T. Nakatsukasa, and K. Yabana, Phys. Rev. C 84, 021302 (2011).

[58] J. Dobaczewski, H. Flocard, and J. Treiner, Nucl. Phys. A 422, 103 (1984).

[59] R. Mohan, M. Danos, and L. Biedenharn, Phys. Rev. C 3, 1740 (1971).

[60] Y. Suzuki, K. Ikeda, and H. Sato, Prog. Theor. Phys. 83, 180 (1990)

[61] P. van Isacker, M. A. Nagarajan, and D. D. Warner, Phys. Rev. C 45, R13 (1992).

[62] P.-G. Reinhard and W. Nazarewicz, Phys. Rev. C 81, 051303(R) (2010).

[63] T. Inakura, T. Nakatsukasa, and K. Yabana, Phys. Rev. C 88, 051305 (2013).

[64] P. Ring and P. Schuck, The Nuclear Many-Body Problems, Texts and Monographs in Physics (Springer-Verlag, New York, 1980).

[65] M. Brack, J. Damgaard, A. S. Jensen, H. C. Pauli, V. M. Strutinsky, and C. Y. Wong, Rev. Mod. Phys. 44, 320 (1972)

[66] N. Tajima, S. Takahara, and N. Onishi, Nucl. Phys. A 603, 23 (1996).

[67] T. Nakatsukasa and K. Yabana, Phys. Rev. C 71, 024301 (2005).

[68] N. Paar, D. Vretenar, E. Khan, and G. Colò, Rep. Prog. Phys. 70, 691 (2007).

[69] P.-G. Reinhard and H. Flocard, Nucl. Phys. A 584, 467 (1995).

[70] B. L. Berman and S. C. Fultz, Rev. Mod. Phys. 47, 713 (1975).

[71] A. Bohr and B. R. Mottelson, Nuclear Structure, Vol. II (W.A. Benjamin, New York, 1975).

[72] M. N. Harakeh and A. van der Woude, Giant Resonances, Oxford Studies in Nuclear Physics Vol. 24 (Oxford University Press, Oxford, 2001).

[73] K. Yoshida and T. Nakatsukasa, Phys. Rev. C 83, 021304 (2011).

[74] D. Peña Arteaga, E. Khan, and P. Ring, Phys. Rev. C 79, 034311 (2009). 